\title{
Effect of contrast medium on treatment modalities planned with different photon beam energies: a planning study
}

\author{
Manindra Bhushan ${ }^{1,2}$, Deepak Tripathi², Girigesh Yadav ${ }^{1}$, Lalit Kumar ${ }^{1}$, Abhinav Dewan ${ }^{1}$, Sarthak Tandon ${ }^{1}$, \\ Gourav Kumar ${ }^{1}$, Inderjit Kaur Wahi ${ }^{1}$, Munish Gairola ${ }^{1}$ \\ ${ }^{1}$ Division of Medical Physics and Department of Radiation Oncology, Rajiv Gandhi Cancer Institute and Research Centre, New Delhi, India \\ ${ }^{2}$ Amity School of Applied Sciences, Amity University (AUUP), NOIDA, Mumbai, India
}

\begin{abstract}
Background: Routinely, patient's planning scans are acquired after administration of iodinized contrast media but they will be treated in the absence of that. Similarly, high energy photons have a better penetrating power, while low energy photons will result in tighter dose distribution and negligible neutron contamination. The aim of the study was to investigate a suitable photon beam energy in the presence of intravenous contrast medium.

Materials and methods: An indigenously made original-contrast (OC) phantom was mentioned as virtual-contrast (VC) and virtual-without-contrast (VWC) phantom were generated by assigning the Hounsfield Units (HU) to different structures. Intensity-modulated (IMRT) and volumetric-modulated-arc (VMAT) plans were generated as per criteria of the TG-119 protocol. Results: It was observed that the maximum dose to the spinal cord was better with 6 mega-voltage (MV) in IMRT. The coverage of Prostate PTV (PR PTV) was similar with all the photon energies and was comparable with TG-119, except for original-contrast (OC) phantom using the VMAT technique. Homogeneity-index (HI) was comparatively better for VMAT plans.

Conclusion: The contrast CT images lower the dose to targets. IMRT or VMAT plans, generated on such CT images will be delivered with higher doses than evaluated. However, the overdose remains non-significant.
\end{abstract}

Key words: contrast-enhanced CT; AAPM TG119; integral dose

Rep Pract Oncol Radiother 2021;26(5):688-711

\section{Introduction}

The aim of radiotherapy is to deliver maximum effective unified dose to tumour target while sparing the nearby critical structures or keeping them within their tolerances. As per literature, one should use higher photon beam energy for deeper targets, like carcinoma of cervix and carcinoma prostate. It is generally seen that lateral separation of such patients remains approximately $20 \mathrm{~cm}$ or more. As a conventional approach of treatment of such cases, the combination of $6 \mathrm{MV}$ and $15 \mathrm{MV}$ is used in the box-field technique. The drawback of such technique is less sparing of the organs-at-risk, i.e. the bladder and rectum. In the same manner, achieving the dose to the spinal cord in head-and-neck cases or carcinoma lung is always challenging.

The evolution of technology has changed the scenario by facilitating the planner to use a 3-dimensional conformal-radiation-therapy (3DCRT) approach. The definition of conformality includes the usage of tomographic images for treatment 
planning. Routinely, the tomographic images are acquired after administration of iodinized intravenous contrast media to patients. Contrast enhanced tomographic images help in better delineation of planning target volumes and organs-at-risk.

Although the treatment plans will be generated on contrast-enhanced computed-tomography (CT) images, the treatment will be delivered in the absence of contrast media. This causes a debate among practitioners about the application of intravenous contrast. Undoubtedly, the presence of contrast medium in planning CT enhances the recognition of a tumour target and improves the ability to provide an accurate delineation of structures but conducting a comprehensive study to evaluate the effect of intravenous contrast on the treatment planning is a necessity.

Conformal radiation therapy is widely accepted in the treatment of carcinoma of cervix which is the fourth most common cancer among women [1]. As per report of the World Health Organization (WHO) of 2018, an estimated 570,000 women were diagnosed with cervical cancer worldwide and approximately 300,000 women died from the disease [2]. Radiation related toxicities are one of the reasons for these causalities.

Intensity-modulated-radiation-therapy (IMRT) has come up with major benefits when compared with 3DCRT as this technique facilitates the planner to modify the photon beam fluence in the optimum manner [3]. The upgrade of technology has brought the features of continuous motion of the gantry and MLC to deliver treatment plans with a variable dose rate. This technique is known as 'volumetric-modulated-arc-therapy (VMAT)'.

Generally, the selection of photon beam energy depends on target volume, patient physical size and tumour depth but $6 \mathrm{MV}$ photon beam is a choice of treatment in most of the clinical sites with different advanced modalities. High energy photons have better penetrating power, skin sparing effect and lesser normal tissue doses while the low energy photons will have narrow penumbra and will result in tighter dose distribution around the target, minimum dose to nearby structures, negligible neutron contamination, minimum head leakage and internal scatter.

Higher energy photons tend to increase the risk of induction of secondary malignancies because of greater collimator leakage and scatter, patient scatter and photo-neutron production [4] whereas the lower energy photons deposit higher doses near beam entry regions and the treatment plan requires greater number of fields, beam segments and monitor units. These adverse skin reactions are major concern in deep seated targets [5]. It has been observed that monitor units and treatment time increases in IMRT as compared to VMAT, which leads to concern about fatal risk of secondary malignancies [6]. It is reported that the risk increases up to $3.4 \%$ with $15 \mathrm{MV}$ photons whereas it varies around 1\%-3\% with $6 \mathrm{MV}$ photon beams [7]. Therefore, the evaluation of choice of optimum photon energy is also mandatory in the ambience of contrast medium.

Hence, the present study focuses on investigating the best suitable photon beam energy in the presence of intravenous iodized contrast medium keeping the hypothesis that contrast media remain insensitive to treatment technique and beam energy.

\section{Materials and methods}

\section{Phantom preparation}

An indigenously made contrast phantom was used for the study. Phantom was made using Perspex sheets (water equivalent material) and water was poured inside to mimic the patient body. A sleeve was provided to place the ionization chamber at the treatment isocentre. Cylindrical chamber (Active volume: $0.125 \mathrm{cc}$ ) was used to evaluate the dose to the isocentre. A vial filled with iodinized contrast, used for patient CT scan, was placed around the ionization chamber sleeve to generate the effect of contrast media. This phantom is called an 'original-contrast (OC)' phantom in the present study as shown in Figure 1 and was scanned on our departmental CT scanner (Somatom Sensation Open, Siemens Medical Solution, Germany). The slice thickness was kept as $0.3 \mathrm{~cm}$ and the scan was transferred to Somavision workstation (Varian Medical Systems) in a dicom format. The similar phantom was created using software and Housnfield Units (HU) were assigned to different structures like phantom walls, filled water, contrast vial and ionization chamber sleeve with active volume as shown in Figure 2. This phantom was utilized as 'virtual contrast (VC)' phantom. Additionally, the contrast vial was assigned the $\mathrm{HU}$ of water and the same phantom was represented as 'virtual-without-contrast (VWC)' phantom. 


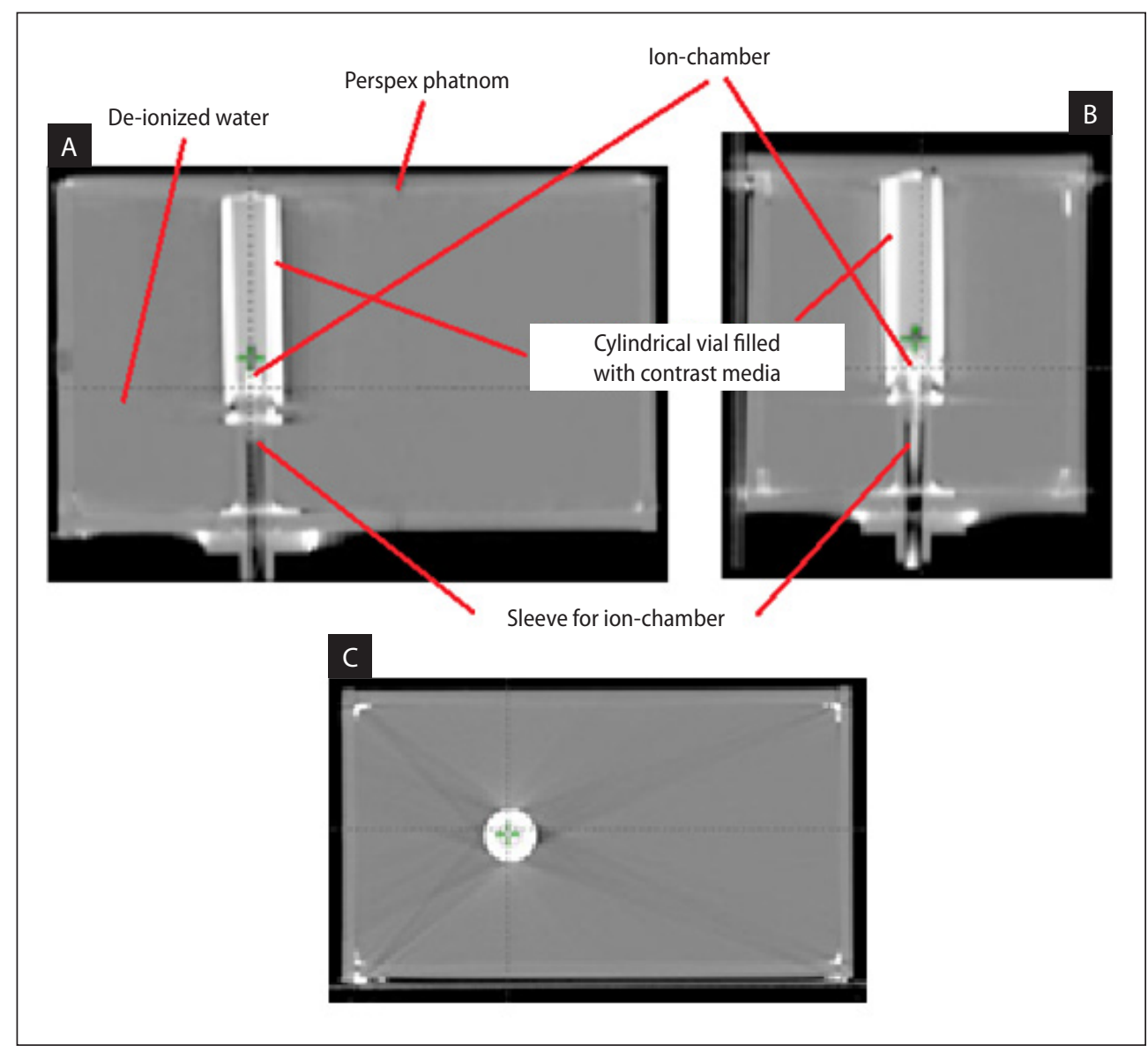

Figure 1. Preparation of phantom. A. Coronal view. B. Sagittal view. C. Axial view

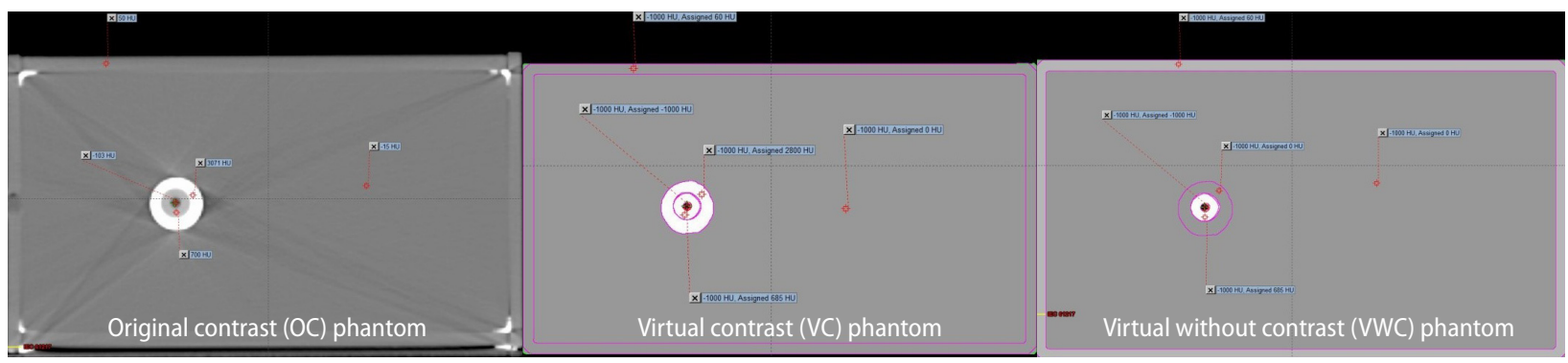

Figure 2. Assigned Housnfield Units (HU) in different parts of phantoms

\section{Contouring}

The structure set provided in recommendations of task group No. 119 of the American Association of Physicists in Medicine (AAPM) was imported from the AAPM website i.e. www.aapm.org and used for planning. This structure set contains four test targets, i.e. Mock head-and-neck, C-shaped target, multi-target (superior-centre-inferior; cylindrical structure of
$2 \mathrm{~cm}$ radius and $4 \mathrm{~cm}$ length each) and mock prostate target. Avoidance structures were the bilateral parotids, spinal cord in mock head neck target; bladder, rectum in mock prostate target and a cylindrical core $(2 \mathrm{~cm}$ diameter with a $0.5 \mathrm{~cm}$ gap between the target and core) in a C-shaped target. All the test target cases, as shown in Figure 3, were imported to all three phantoms for generating the plans. 


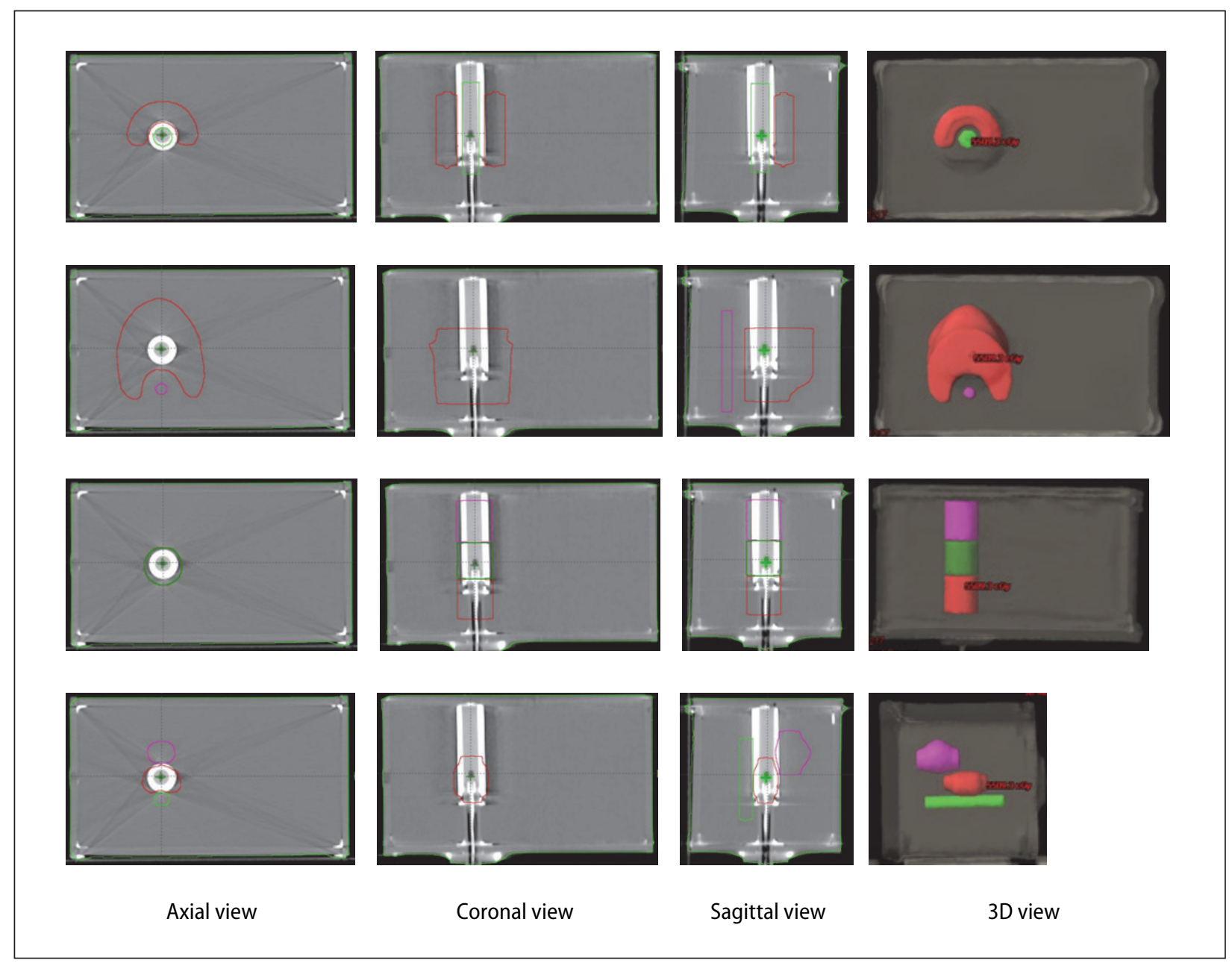

Figure 3. Test targets as per the TG-119 of the American Association of Physicists in Medicine (AAPM TG119) recommendations. A. C-shaped target; B. Head and neck test target; C. Multi-target; D. Prostate test target

\section{Treatment planning}

Treatment plans were generated on Eclipse (Varian Medical Systems, Version 11.0) treatment planning system (TPS) and were delivered on TrueBeam (Varian Medical Systems, Palo Alto, CA, USA; Version 1.6) linear accelerator (linac). Eclipse TPS uses Progressive Resolution Optimization (PRO) algorithm for optimizing the plans and calculates the same using the Analytical Anisotropic Algorithm (AAA) after leaf-motion-calculation. TrueBeam linac is equipped with three flattened photon beam energies, i.e. $6 \mathrm{MV}, 10 \mathrm{MV}, 15 \mathrm{MV}$, and two flattening-filter-free (FFF) energies, i.e. 6 FFF \& 10 FFF. Removal of a flattening filter from the beam path increases the dose rate up to 1400 $\mathrm{MU} / \mathrm{min}$ and $2400 \mathrm{MU} / \mathrm{min}$ for $6 \mathrm{FFF}$ and $10 \mathrm{FFF}$ photon beam, respectively. Multi-leaf-collimator (MLC) are attached at a tertiary level in Varian's TrueBeam linac and are characterized by spatial resolution of $0.25 \mathrm{~cm}$ at the isocentre for the central 32 pairs of leaves and $0.5 \mathrm{~cm}$ in the outer 28 pairs of leaves.

IMRT plans were generated with gantry angles $0^{\circ}, 51^{\circ}, 102^{\circ}, 153^{\circ}, 204^{\circ}, 255^{\circ}$ and $306^{\circ}$ with collimator $/$ couch $0^{\circ}$ for all the test cases. However, two full-arcs ranging from $181^{\circ}$ to $179^{\circ}$ clockwise and anti-clockwise, were used in VMAT plans.

\section{Comparative evaluation}

The plans were generated as per criteria of the TG 119 protocol and a comparative evaluation was done by qualitative as well as quantitative methods. The plans were evaluated for different test cases on the following parameters - Table 1 .

Plan quality indices, like conformity index and homogeneity index, were also evaluated for selected test cases using the ICRU-83 protocol and the following formula: 
Table. 1. Parameters in the plans evaluated for different test cases

\begin{tabular}{|c|c|c|c|c|c|c|c|c|c|c|c|c|}
\hline Test target & Parameter/stucture & $\mathbf{D}_{99 \%}$ & $\mathrm{D}_{98 \%}$ & $\mathrm{D}_{95 \%}$ & $\mathbf{D}_{90 \%}$ & $\mathbf{D}_{50 \%}$ & $\mathrm{D}_{30 \%}$ & $\mathbf{D}_{20 \%}$ & $\mathbf{D}_{10 \%}$ & $\mathbf{D}_{5 \%}$ & $\mathbf{D}_{2 \%}$ & $\mathbf{D}_{\max }$ \\
\hline \multirow{3}{*}{$\begin{array}{l}\text { Mock } \\
\text { Head neck }\end{array}$} & HN PTV & $x$ & $x$ & & $x$ & $x$ & & $x$ & & & $x$ & \\
\hline & Cord & & & & & & & & & & & $x$ \\
\hline & Bilateral Parotids & & & & & $x$ & & & & & & \\
\hline \multirow{3}{*}{$\begin{array}{l}\text { Mock } \\
\text { multi-target }\end{array}$} & PTV Centre & $x$ & & & & & & & $x$ & & & \\
\hline & PTV Superior & $x$ & & & & & & & $x$ & & & \\
\hline & PTV Inferior & $x$ & & & & & & & $x$ & & & \\
\hline \multirow{2}{*}{$\begin{array}{l}\text { Mock } \\
\text { C-shaped }\end{array}$} & CS PTV & & $x$ & $x$ & & $x$ & & & $x$ & & $x$ & \\
\hline & Core & & & & & & & & & $x$ & & \\
\hline \multirow{3}{*}{$\begin{array}{l}\text { Mock } \\
\text { prostate }\end{array}$} & PR PTV & & $x$ & $x$ & & $x$ & & & & $x$ & $x$ & \\
\hline & Bladder & & & & & & $x$ & & $x$ & & & \\
\hline & Rectum & & & & & & $x$ & & $x$ & & & \\
\hline
\end{tabular}

PTV - planning target volume; CS - C-shaped; PR — prostate

Conformity Index (CI98) [8]:

Volume of $98 \%$ isodose curve (in cc)/ /Volume of PTV (in cc)

Homogeneity Index (HI) [9]:

$$
\left(D_{2 \%}-D_{98 \%}\right) / D_{50 \%}
$$

Dose spillage to nearby normal tissues remains a concern for the planners and, hence, the integral dose to normal tissues [10] for the structure 'body-PTV', was calculated using the following formula:

Normal-tissue-integral-dose (NTID): Mean dose (in Gy) $x$ Volume of structure (in cc)

\section{Statistical analysis}

Data collected was validated with other published data and AAPM TG 119 values were taken as a standard. Inter-comparison test was performed to analyze the data to find out statistically significant results for better interpretation. The formula used was:

\section{Percentage deviation $=($ Measured value $) /$ /(AAPM TG 119 limiting value)}

\section{Results}

Dosimetric deviation of IMRT and VMAT plans in VWC phantom with published studies for TG 119 recommendations with $6 \mathrm{MV}$ photon beam energy was calculated and tabulated in Table 2 . It is mentioned that the present study in without con- trast phantom has significant correlation with other published data which was performed in a similar ambience, i.e. in the absence of contrast media. This experimental data was analyzed to make a resemblance with published data and made a comparison with results of AAPM TG 119 [11], Mynampati et al. [12], Nithya et al. [13], Lalit et al. [14] and Kaushik et al. [15].

Further, the plans were generated with varying photon beam energies mentioned above, and the results were tabulated in Table 3. The dose coverage (95\% of maximum prescribed dose) to PTV for different test cases in all the three views (axial, coronal and sagittal) are shown in Figure 4 for different techniques and photon beam energies in selected phantoms.

\section{Mock head and neck case}

For the head and neck test case, the HN PTV target coverage to $99 \%$ of volume was in comparison with TG 119 results. It was observed that the maximum dose to the spinal cord was better with $6 \mathrm{MV}$ photon beam energy in the IMRT technique and tabulated in Table 3 and Table 4.

\section{Mock multi-target case}

In this case, all the parameters of target coverage were achieved, except for the inferior target in VMAT cases with all energies. Also, the $\mathrm{D}_{10 \%}$ for the central target was not achieved in most of the contrast phantoms. Mynampati et al. [12] and Ezzell et al. [11] also reported the higher $\mathrm{D}_{10 \%}$ for the central target in their studies. However, the 


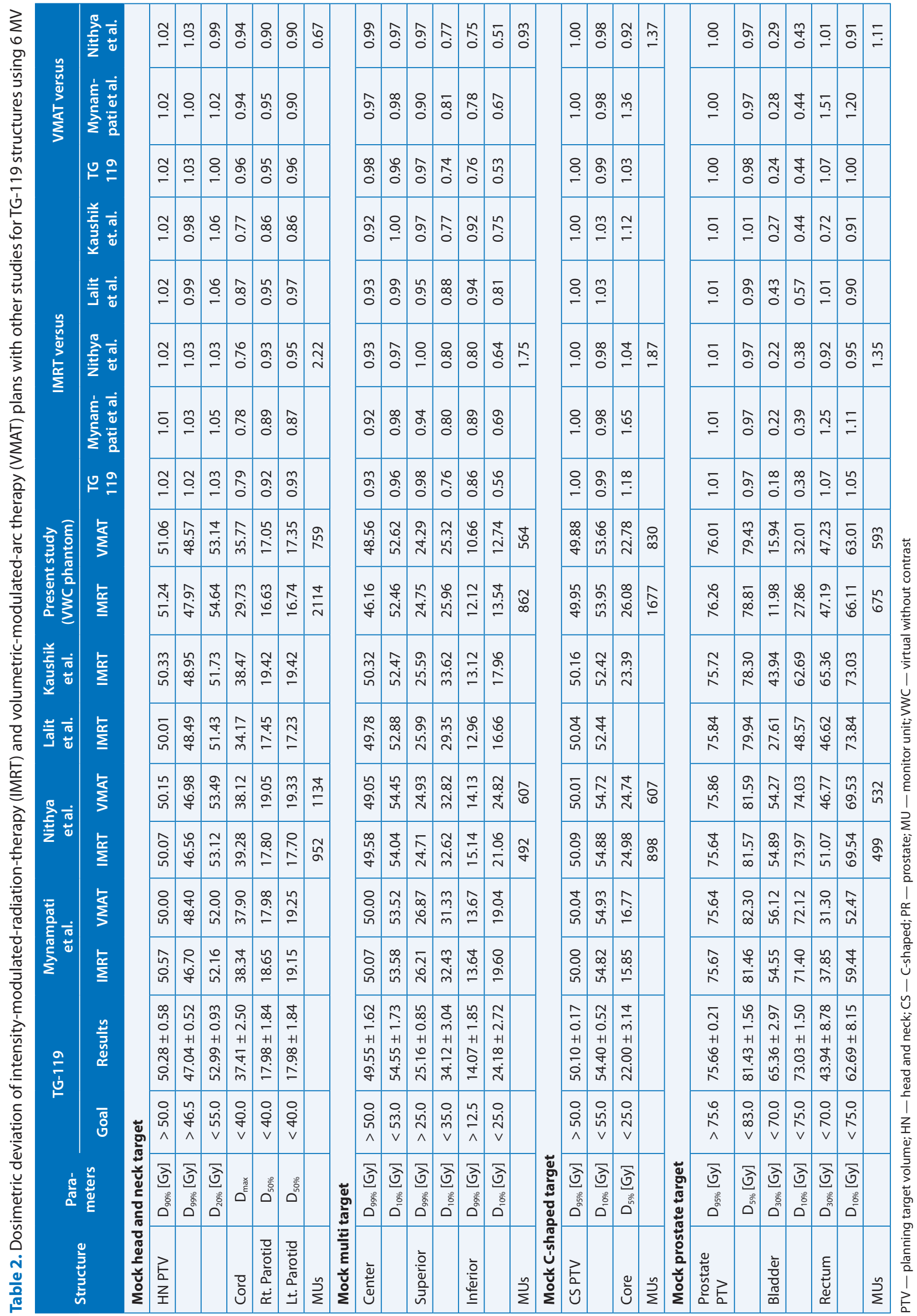




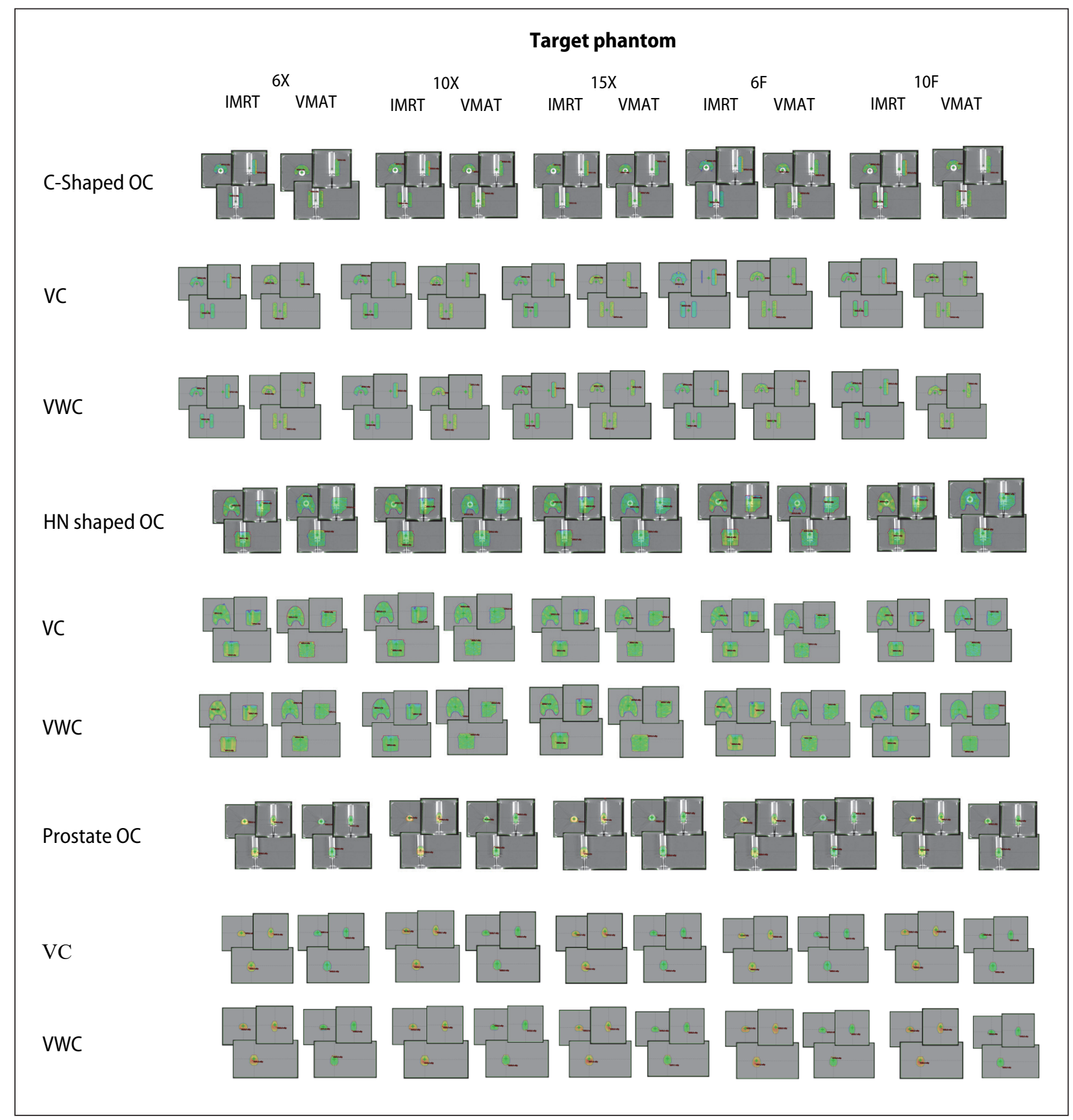

Figure 4. PTV dose coverage ( $95 \%$ of maximum prescribed dose) of test cases for different techniques and photon beam energies in different phantoms. OC — original contrast; VC — virtual contrast; VWC — virtual without contras

present data demonstrated better results for $\mathrm{D}_{10 \%}$ for the inferior target for all the available energies in both techniques as compared to the TG 119 result (24.18 Gy).

\section{Mock C-shaped target case}

For the C-shaped target case, the dose to 95\% volume of CS PTV (i.e. C-shaped Planning Target Volume) was comparable with all the photon beam energies and phantoms and was closer to values achieved by TG 119 . The dose received by $5 \%$ volume of the 'core' structure was observed as exceeded in IMRT plans from the prescribed limit $\left(\mathrm{D}_{5 \%}<25 \mathrm{~Gy}\right)$ and shown an upward trend with increasing photon beam energies. The results were tabulated in Table 5 and Table 6.

\section{Mock prostate case}

For the prostate test target, the coverage of PR PTV (i.e. Prostate PTV) was similar with all the 


\begin{tabular}{|c|c|c|c|c|c|c|c|c|c|c|c|c|c|c|c|c|c|c|c|}
\hline & 낭 & $\begin{array}{l}\text { t } \\
\text { Oे } \\
\text { in }\end{array}$ & $\underset{\substack{M \\
\stackrel{\infty}{\sigma}}}{M}$ & $\begin{array}{l}\tilde{O} \\
\stackrel{f}{f}\end{array}$ & $\overline{\vec{P}}$ & $\begin{array}{l}\infty \\
\\
\end{array}$ & $\begin{array}{l}\infty \\
\stackrel{\infty}{*} \\
\stackrel{n}{n}\end{array}$ & $\begin{array}{l}\stackrel{\infty}{\circ} \\
\stackrel{\sim}{\sim}\end{array}$ & $\begin{array}{l}\stackrel{0}{0} \\
\stackrel{6}{6}\end{array}$ & 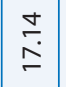 & ڤ̊̊ి & & $\begin{array}{l}\stackrel{t}{\Delta} \\
\dot{g}\end{array}$ & 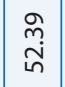 & $\begin{array}{l}0 \\
\stackrel{+}{\sim} \\
\stackrel{+}{~}\end{array}$ & 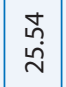 & $\stackrel{\underset{N}{N}}{\stackrel{N}{I}}$ & $\begin{array}{l}\hat{n} \\
\stackrel{n}{n}\end{array}$ & ণ \\
\hline & $\frac{\frac{1}{4}}{\frac{1}{6}}$ & $\frac{m}{\dot{b}}$ & $\begin{array}{l}\bar{\infty} \\
\infty \\
o \\
o\end{array}$ & $\begin{array}{c}\tilde{\infty} \\
\stackrel{f}{f}\end{array}$ & $\begin{array}{l}\stackrel{n}{n} \\
\tilde{n} \\
n\end{array}$ & $\begin{array}{l}\bar{F} \\
\stackrel{f}{n}\end{array}$ & $\begin{array}{l}\stackrel{g}{g} \\
\stackrel{\leftrightarrow}{n}\end{array}$ & $\begin{array}{l}\text { ñ } \\
\stackrel{\leftrightarrow}{\text { N }}\end{array}$ & 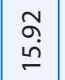 & $\begin{array}{l}\infty \\
0 \\
0\end{array}$ & $\stackrel{\infty}{\stackrel{\sim}{N}}$ & & \begin{tabular}{l}
$\underset{\sim}{\tilde{\sigma}}$ \\
\multirow{\sigma}{*}{}
\end{tabular} & \begin{tabular}{|c|}
$\stackrel{a}{i}$ \\
$i$ \\
$i$
\end{tabular} & $\begin{array}{l}\tilde{m} \\
\stackrel{\dot{j}}{\sim}\end{array}$ & $\begin{array}{l}m \\
\stackrel{m}{\omega} \\
\dot{\omega}\end{array}$ & 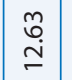 & 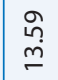 & $\stackrel{\nabla}{m}$ \\
\hline$\frac{\pi}{0}$ & 峞 & $\begin{array}{l}\widehat{c} \\
0 \\
i n \\
i n\end{array}$ & 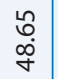 & 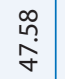 & $\underset{\sim}{\sim}$ & $\begin{array}{l}\tilde{O} \\
\stackrel{+}{n}\end{array}$ & $\begin{array}{l}\tilde{\alpha} \\
\dot{\mathfrak{n}}\end{array}$ & $\begin{array}{l}\text { ๙े } \\
\text { নे }\end{array}$ & 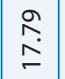 & 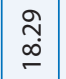 & ટ્తి & & $\begin{array}{l}\bar{f} \\
\dot{b}\end{array}$ & $\begin{array}{c}\sim \\
i \\
i n\end{array}$ & 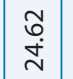 & \begin{tabular}{l|}
$\bar{\infty}$ \\
$\dot{\sim}$ \\
$\dot{\sim}$
\end{tabular} & $\underset{\mathfrak{I}}{\stackrel{\mathfrak{I}}{ }}$ & $\begin{array}{l}\stackrel{\propto}{ } \\
\stackrel{\sim}{p}\end{array}$ & $\grave{\nwarrow}$ \\
\hline & 즘 & $\underset{i}{\bar{i}}$ & 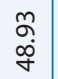 & \begin{tabular}{l}
$\stackrel{8}{\Im}$ \\
\multirow{f}{*}{}
\end{tabular} & 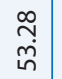 & $\begin{array}{l}\text { J } \\
\dot{\forall} \\
\text { in }\end{array}$ & 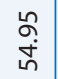 & $\begin{array}{c}\infty \\
\stackrel{\infty}{0} \\
\stackrel{m}{m}\end{array}$ & $\begin{array}{l}\stackrel{\bullet}{\circ} \\
\stackrel{-}{二}\end{array}$ & $\begin{array}{l}\underset{\sim}{\infty} \\
\stackrel{\infty}{\infty}\end{array}$ & $\begin{array}{l}\infty \\
\stackrel{\infty}{\circ}\end{array}$ & & $\begin{array}{l}\tilde{\sigma} \\
\delta \\
\delta\end{array}$ & \begin{tabular}{|c|c|} 
\\
$\infty$ \\
$i$ \\
\end{tabular} & $\begin{array}{l}\infty \\
\stackrel{\vdots}{+} \\
\dot{\sim}\end{array}$ & \begin{tabular}{|l|}
$\stackrel{\sim}{\sim}$ \\
$\stackrel{\sim}{S}$
\end{tabular} & 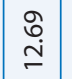 & $\begin{array}{l}\stackrel{a}{ } \\
\dot{m}\end{array}$ & స్ \\
\hline & & $\begin{array}{l}\infty \\
\stackrel{\infty}{0} \\
\stackrel{0}{n}\end{array}$ & 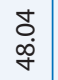 & $\begin{array}{l}\infty \\
\stackrel{6}{6} \\
o\end{array}$ & $\begin{array}{l}\bar{\sigma} \\
\stackrel{\tilde{n}}{n}\end{array}$ & 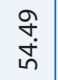 & $\begin{array}{l}\text { gे } \\
\text { in }\end{array}$ & $\frac{\circ}{\circ}$ & $\begin{array}{l}\stackrel{M}{0} \\
\hat{\sigma}\end{array}$ & $\begin{array}{l}\hat{\Omega} \\
\hat{\sigma}\end{array}$ & 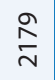 & & $\begin{array}{l}\vec{a} \\
\dot{g}\end{array}$ & 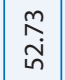 & \begin{tabular}{l}
\multirow{f}{*}{} \\
$\dot{\sim}$
\end{tabular} & \begin{tabular}{|c|}
$\bar{m}$ \\
$\stackrel{i}{i}$
\end{tabular} & $\stackrel{\bar{n}}{\mathfrak{I}}$ & $\begin{array}{l}\stackrel{+}{~} \\
\stackrel{m}{m}\end{array}$ & $\underset{g}{\underline{g}}$ \\
\hline & & $\begin{array}{l}0 \\
m \\
0 \\
i n n\end{array}$ & 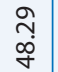 & $\underset{m}{\stackrel{m}{f}}$ & $\begin{array}{l}\infty \\
\stackrel{1}{0} \\
\end{array}$ & 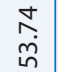 & 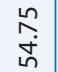 & $\begin{array}{l}\stackrel{n}{n} \\
\hat{\rho} \\
\end{array}$ & 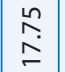 & 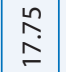 & $\stackrel{\text { L }}{\text { N }}$ & & $\begin{array}{l}\bar{R} \\
\dot{j}\end{array}$ & 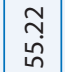 & $\begin{array}{l}m \\
\stackrel{m}{\sim}\end{array}$ & \begin{tabular}{|}
$\stackrel{\sim}{\sim}$ \\
$\underset{\sim}{\sim}$
\end{tabular} & $\begin{array}{c}\tilde{J} \\
\stackrel{m}{=}\end{array}$ & సู & $\underset{\widetilde{\sigma}}{=}$ \\
\hline & & 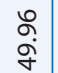 & $\begin{array}{l}\stackrel{\infty}{\stackrel{\sim}{*}} \\
\stackrel{f}{*}\end{array}$ & $\begin{array}{l}\hat{n} \\
\hat{\sigma} \\
\sigma\end{array}$ & $\underset{\substack{\text { g } \\
n}}{ }$ & 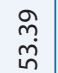 & 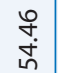 & $\overline{\text { Dे }}$ & $\begin{array}{l}\underset{0}{0} \\
\stackrel{0}{0}\end{array}$ & $\begin{array}{c}\bar{\sigma} \\
\stackrel{n}{n}\end{array}$ & ্ָতি & & $\begin{array}{l}\frac{0}{\dot{g}} \\
\dot{q}\end{array}$ & \begin{tabular}{|l|} 
\\
\\
$ن$ \\
\end{tabular} & 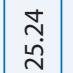 & \begin{tabular}{|l|} 
\\
$\stackrel{0}{0}$ \\
$\stackrel{0}{0}$
\end{tabular} & 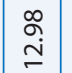 & $\begin{array}{l}\stackrel{g}{\alpha} \\
\stackrel{m}{q}\end{array}$ & 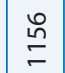 \\
\hline$\frac{5}{\frac{5}{2}}$ & un & $\begin{array}{l}\bar{f} \\
\text { in }\end{array}$ & $\begin{array}{c}\hat{A} \\
\stackrel{\infty}{\sigma}\end{array}$ & $\begin{array}{c}\hat{m} \\
\stackrel{f}{f}\end{array}$ & $\begin{array}{l}\stackrel{n}{\infty} \\
\stackrel{i}{n} \\
\end{array}$ & $\begin{array}{l}\bar{\sigma} \\
\stackrel{\sim}{n}\end{array}$ & 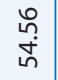 & 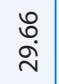 & $\begin{array}{l}\tilde{f} \\
\stackrel{\infty}{\infty}\end{array}$ & $\begin{array}{l}\bar{R} \\
\infty \\
\infty\end{array}$ & 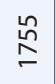 & & $\bar{g}$ & $\begin{array}{l}\stackrel{0}{2} \\
\text { 字 }\end{array}$ & $\mid \begin{array}{l}\stackrel{g}{d} \\
\dot{d}\end{array}$ & 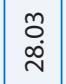 & 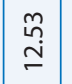 & $\begin{array}{l}\stackrel{a}{m} \\
\stackrel{n}{n}\end{array}$ & $\stackrel{\widehat{o}}{\sigma}$ \\
\hline & & $\begin{array}{l}n 0 \\
0 \\
0 \\
i n\end{array}$ & 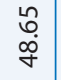 & $\begin{array}{l}\bar{\sigma} \\
\stackrel{f}{f}\end{array}$ & 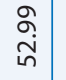 & $\begin{array}{l}\hat{N} \\
\text { in }\end{array}$ & $\begin{array}{l}\bar{N} \\
\stackrel{5}{n}\end{array}$ & 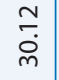 & 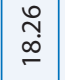 & $\begin{array}{l}\hat{\omega} \\
\infty \\
\infty \\
\infty\end{array}$ & $\stackrel{0}{\stackrel{0}{I}}$ & & $\begin{array}{l}\infty \\
0 \\
\dot{g}\end{array}$ & 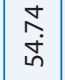 & $\begin{array}{l}o \\
\dot{d} \\
\dot{d}\end{array}$ & \begin{tabular}{|c|}
$\bar{\sim}$ \\
$\infty$ \\
$\sim$
\end{tabular} & $\begin{array}{l}\infty \\
\stackrel{\infty}{+} \\
\stackrel{+}{ \pm}\end{array}$ & $\begin{array}{l}\hat{A} \\
\stackrel{\leftrightarrow}{n}\end{array}$ & $\underset{\mathfrak{m}}{\bar{n}}$ \\
\hline & & $\begin{array}{l}\infty \\
\stackrel{0}{0} \\
\text { in }\end{array}$ & 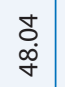 & $\begin{array}{l}\text { aे } \\
\dot{\sigma}\end{array}$ & 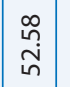 & 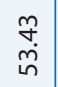 & $\begin{array}{l}J \\
\stackrel{f}{*}\end{array}$ & $\frac{m}{i}$ & $\begin{array}{l}\infty \\
\infty \\
\stackrel{\infty}{0}\end{array}$ & 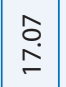 & 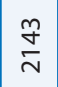 & & $\begin{array}{l}\underset{N}{\sim} \\
\stackrel{f}{f}\end{array}$ & \begin{tabular}{|l|} 
\\
$\stackrel{0}{0}$ \\
$\stackrel{+}{n}$
\end{tabular} & 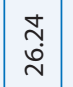 & 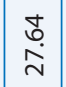 & $\begin{array}{l}\stackrel{\sim}{\infty} \\
\stackrel{\sim}{\sim}\end{array}$ & $\begin{array}{l}\bar{\sigma} \\
\stackrel{+}{5}\end{array}$ & $\stackrel{\circ}{0}$ \\
\hline & & $\begin{array}{c}\bar{b} \\
\text { in }\end{array}$ & 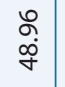 & 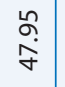 & 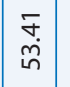 & 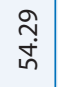 & $\begin{array}{l}\stackrel{m}{\sharp} \\
\text { 出 }\end{array}$ & $\begin{array}{l}\bar{\infty} \\
\stackrel{\sim}{i}\end{array}$ & 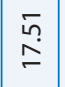 & 总 & 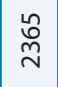 & & 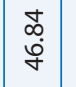 & 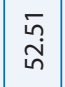 & 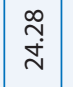 & $\begin{array}{l}\hat{b} \\
\stackrel{\leftrightarrow}{\dot{d}}\end{array}$ & 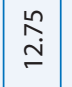 & $\begin{array}{l}\stackrel{\circ}{0} \\
\stackrel{m}{m}\end{array}$ & ऽิ \\
\hline & & $\begin{array}{l}\infty \\
\stackrel{\infty}{0} \\
0 \\
i n n\end{array}$ & 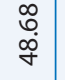 & 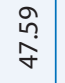 & 芯 & \begin{tabular}{l}
$\stackrel{a}{m}$ \\
\multirow{n}{*}{}
\end{tabular} & 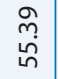 & $\begin{array}{l}\bar{\alpha} \\
\text { ळे }\end{array}$ & $\begin{array}{l}\stackrel{\sim}{\infty} \\
\stackrel{\leftrightarrow}{\sim}\end{array}$ & 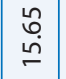 & $\stackrel{\infty}{\stackrel{\infty}{\sim}}$ & & 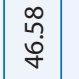 & $\begin{array}{c}0 \\
\text { i⿱ } \\
i n\end{array}$ & $\begin{array}{l}\stackrel{+}{+} \\
\stackrel{+}{\sim}\end{array}$ & \begin{tabular}{l|}
$\bar{\sigma}$ \\
$\stackrel{\text { ja }}{ }$
\end{tabular} & 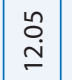 & $\begin{array}{l}\bar{N} \\
\stackrel{m}{n}\end{array}$ & $\stackrel{\circ}{\infty}$ \\
\hline$\frac{\pi}{\frac{\pi}{2}}$ & & $\begin{array}{l}\text { ̊̊. } \\
\text { Oें }\end{array}$ & $\begin{array}{l}\infty \\
\infty \\
\infty \\
\sigma \\
\sigma\end{array}$ & $\begin{array}{l}\bar{\infty} \\
\stackrel{\sim}{\forall}\end{array}$ & 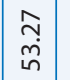 & $\begin{array}{l}m \\
\stackrel{0}{0} \\
\dot{b}\end{array}$ & $\begin{array}{l}\infty \\
\stackrel{\infty}{*} \\
\stackrel{n}{n}\end{array}$ & $\begin{array}{l}\stackrel{8}{0} \\
\stackrel{\dot{m}}{m}\end{array}$ & $\begin{array}{l}\stackrel{\infty}{\circ} \\
\stackrel{\sim}{二}\end{array}$ & $\underset{\infty}{\stackrel{J}{\sim}}$ & $\underset{Ð}{\mathfrak{Z}}$ & & 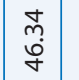 & 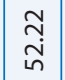 & $\mid \begin{array}{l}\stackrel{\odot}{a} \\
\stackrel{\sim}{\sim}\end{array}$ & $\frac{m}{\dot{d}}$ & $\stackrel{\underset{I}{I}}{\stackrel{I}{I}}$ & $\begin{array}{l}\stackrel{\sim}{\infty} \\
\stackrel{m}{\sim}\end{array}$ & $\hat{\infty}$ \\
\hline & & $\underset{\stackrel{n}{\sim}}{\stackrel{\text { in }}{n}}$ & $\begin{array}{l}\stackrel{a}{a} \\
\dot{g}\end{array}$ & $\begin{array}{l}\tilde{O} \\
\stackrel{\infty}{\sigma}\end{array}$ & $\begin{array}{l}\stackrel{n}{n} \\
\stackrel{n}{n}\end{array}$ & $\begin{array}{l}\text { సે } \\
\text { ָे }\end{array}$ & $\stackrel{m}{i n}$ & $\overline{\dot{m}}$ & 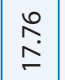 & $\begin{array}{l}\stackrel{\infty}{\mathbf{D}} \\
\infty \\
\sim\end{array}$ & $\begin{array}{l}\stackrel{\sim}{0} \\
\propto \\
\sim\end{array}$ & & 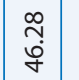 & 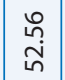 & $\mid \begin{array}{l}\stackrel{g}{+} \\
\dot{d}\end{array}$ & \begin{tabular}{|l|}
$\stackrel{n}{b}$ \\
$\dot{d}$
\end{tabular} & 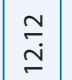 & $\begin{array}{l}\hat{\sigma} \\
\stackrel{p}{~}\end{array}$ & 옹 \\
\hline & & 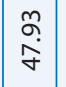 & $\begin{array}{l}\infty \\
\stackrel{\infty}{0} \\
\stackrel{\infty}{\sigma}\end{array}$ & $\stackrel{\stackrel{n}{n}}{\stackrel{n}{n}}$ & 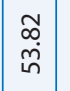 & 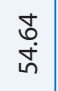 & $\begin{array}{l}\hat{h} \\
\hat{n} \\
\hat{n}\end{array}$ & $\begin{array}{l}\stackrel{m}{a} \\
\stackrel{N}{N}\end{array}$ & 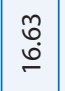 & $\begin{array}{l}2 \\
6 \\
0\end{array}$ & $\frac{⿱ 亠}{\bar{N}}$ & & 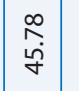 & $\begin{array}{l}0 \\
\text { I } \\
\text { in }\end{array}$ & $\mid \begin{array}{l}2 \\
+ \\
\dot{d}\end{array}$ & 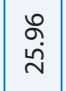 & 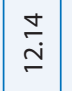 & 岕 & ণ్ \\
\hline & 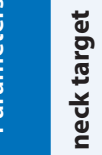 & 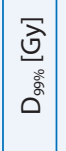 & 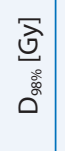 & 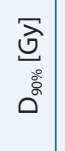 & 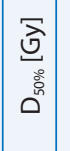 & 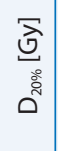 & $\begin{array}{l}\text { 勇 } \\
0^{2}\end{array}$ & 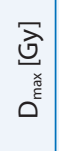 & 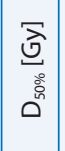 & 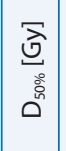 & & & 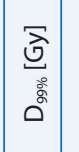 & 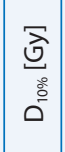 & 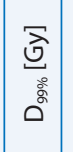 & 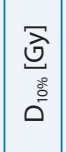 & 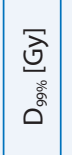 & 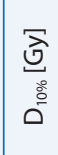 & \\
\hline$\underline{Z}$ & 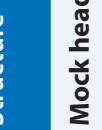 & & & & & & & 힘 & 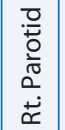 & 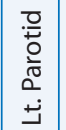 & $\stackrel{\vec{\Sigma}}{\Sigma}$ & 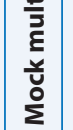 & & $\bar{\Psi}$ & $\begin{array}{l}\text { 일 } \\
\stackrel{3}{\Xi} \\
\text { ज }\end{array}$ & 产 & 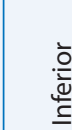 & 产 & $\vec{\Sigma}$ \\
\hline
\end{tabular}




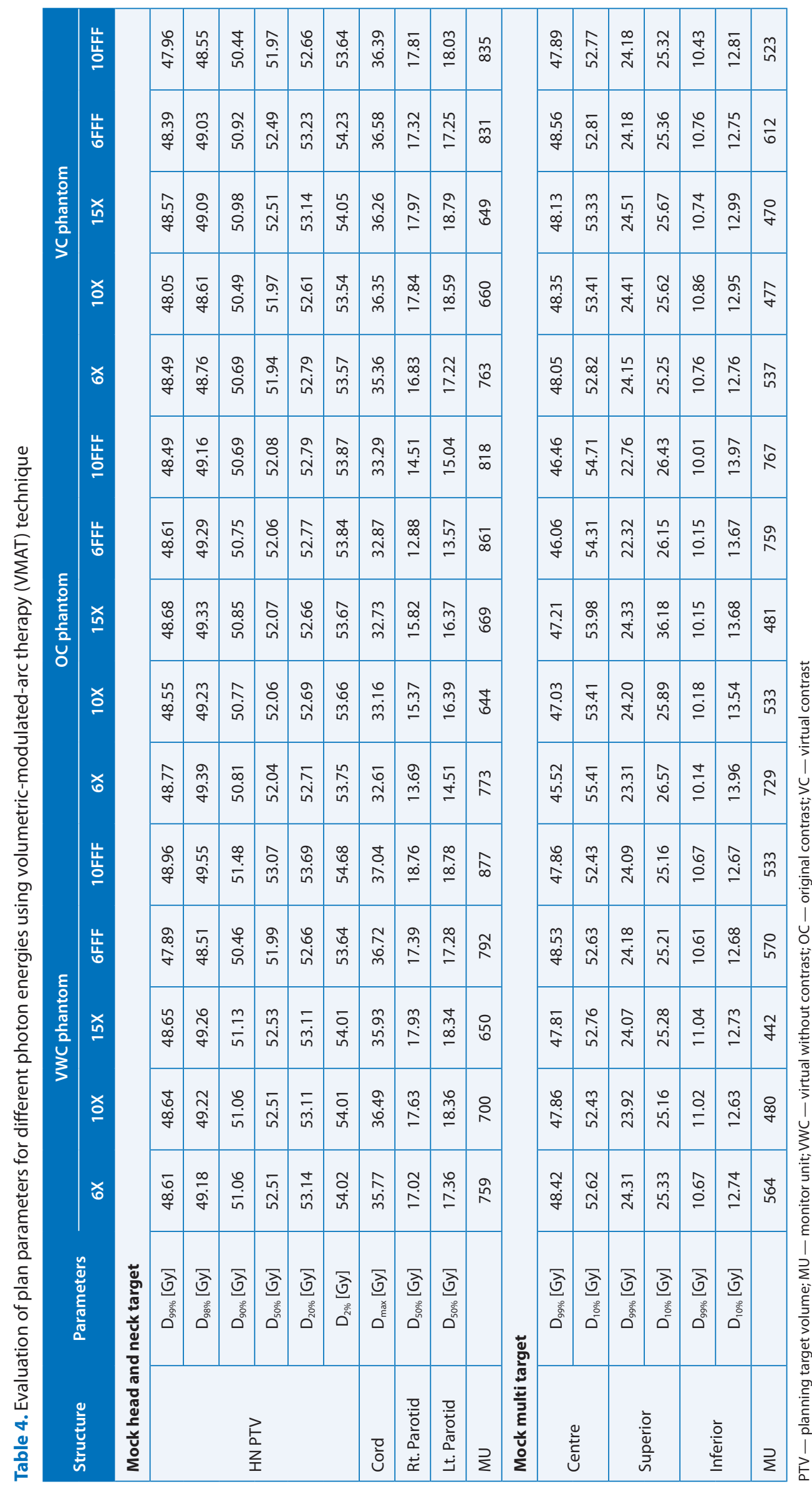




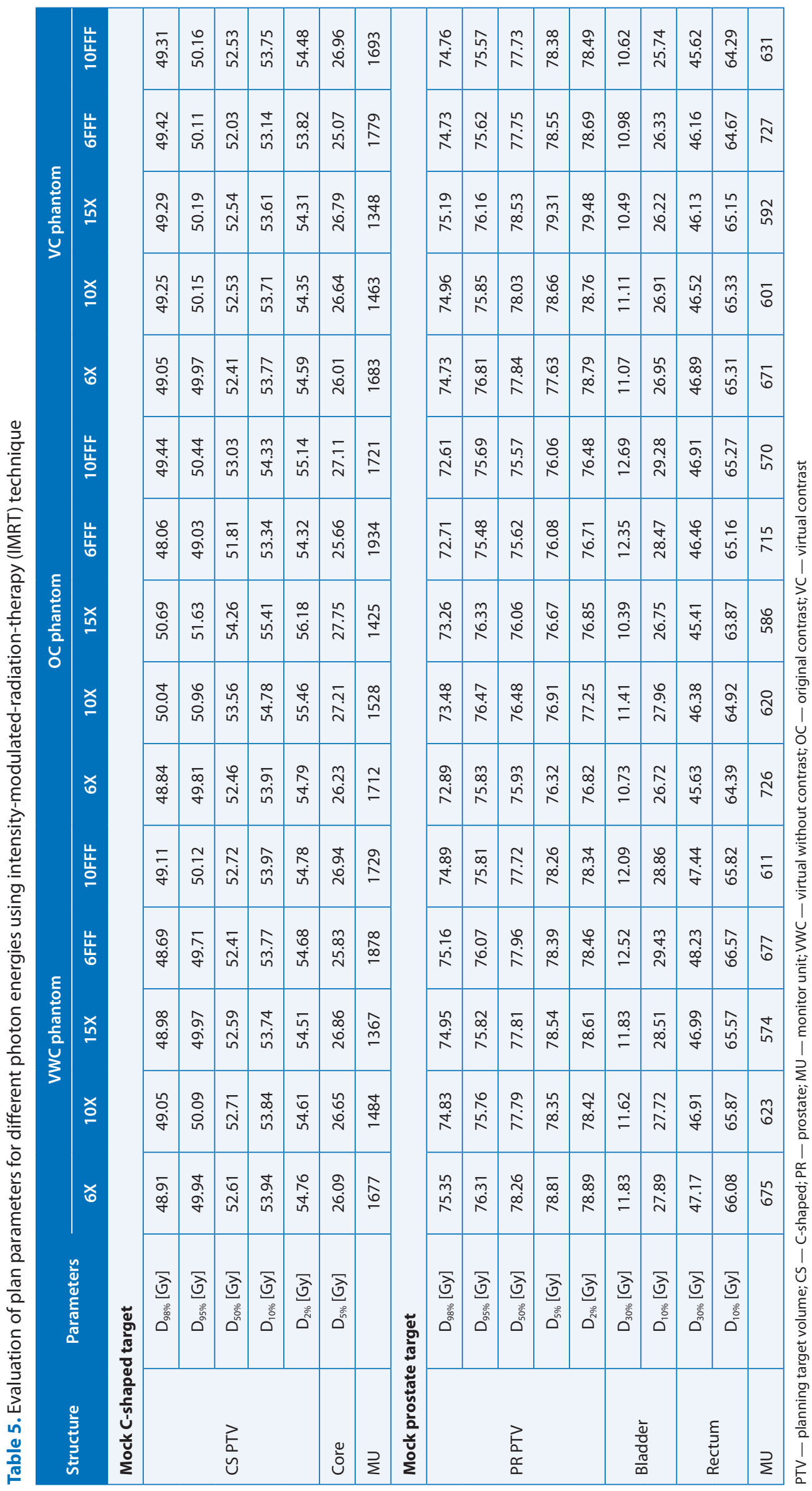




\begin{tabular}{|c|c|c|c|c|c|c|c|c|c|c|c|c|c|c|c|c|c|c|c|}
\hline & $\frac{1}{\frac{1}{4}}$ & $\begin{array}{l}n \\
\text { o. } \\
\alpha \\
\sigma\end{array}$ & $\begin{array}{l}\hat{\hat{q}} \\
\dot{g}\end{array}$ & $\bar{i}$ & 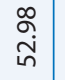 & $\underset{\stackrel{q}{\vec{n}}}{\mathrm{n}}$ & $\underset{\stackrel{N}{N}}{\stackrel{N}{n}}$ & 命 & & $\begin{array}{l}\hat{A} \\
\dot{f}\end{array}$ & $\begin{array}{c}\stackrel{\Omega}{2} \\
\stackrel{n}{n}\end{array}$ & $\begin{array}{l}\stackrel{a}{\infty} \\
\stackrel{1}{\wedge}\end{array}$ & $\begin{array}{l}\stackrel{\sim}{n} \\
\stackrel{\Omega}{n}\end{array}$ & $\begin{array}{l}\bar{\sigma} \\
\stackrel{i}{R}\end{array}$ & $\begin{array}{l}\stackrel{g}{+} \\
\dot{J}\end{array}$ & $\begin{array}{l}m \\
\infty \\
\stackrel{\alpha}{a} \\
\grave{N}\end{array}$ & $\begin{array}{l}\stackrel{m}{\mathfrak{b}} \\
\dot{b}\end{array}$ & $\begin{array}{l}\hat{b} \\
\text { రิ }\end{array}$ & 会 \\
\hline & $\frac{\underline{4}}{\frac{1}{6}}$ & 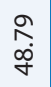 & $\begin{array}{l}\hat{n} \\
\text { ga }\end{array}$ & $\underset{\substack{\sim \\
\text { in }}}{ }$ & $\begin{array}{l}8 \\
\ddot{i} \\
\end{array}$ & $\begin{array}{l}\bar{\sigma} \\
\stackrel{\sim}{n}\end{array}$ & 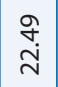 & $\stackrel{\tilde{n}}{n}$ & & $\begin{array}{l}\stackrel{t}{0} \\
\dot{i}\end{array}$ & $\begin{array}{l}\infty \\
\stackrel{2}{2} \\
\stackrel{n}{n}\end{array}$ & 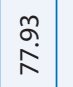 & $\begin{array}{l}\stackrel{0}{1} \\
\text { হ̦ }\end{array}$ & $\begin{array}{c}\hat{b} \\
\stackrel{i}{R}\end{array}$ & $\underset{\substack{r \\
\dot{m}}}{ }$ & 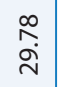 & $\begin{array}{l}\infty \\
\stackrel{2}{b} \\
\dot{y}\end{array}$ & 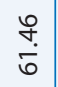 & ? \\
\hline$\frac{\text { s] }}{\frac{5}{2}}$ & 屈 & 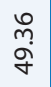 & $\bar{c}$ & 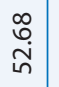 & $\stackrel{i n}{i n}$ & 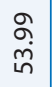 & $\begin{array}{l}n \\
\stackrel{n}{\mathbf{N}} \\
\mathbf{n}\end{array}$ & 峁 & & $\begin{array}{l}\bar{m} \\
\stackrel{p}{N}\end{array}$ & 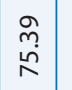 & $\begin{array}{l}\stackrel{n}{\alpha} \\
\stackrel{1}{R}\end{array}$ & $\begin{array}{c}\bar{n} \\
\text { ñ }\end{array}$ & $\begin{array}{l}\hat{\infty} \\
\stackrel{i}{R}\end{array}$ & $\begin{array}{l}\hat{n} \\
\stackrel{\text { h}}{n}\end{array}$ & 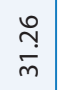 & $\begin{array}{l}\bar{\alpha} \\
\dot{q} \\
\dot{q}\end{array}$ & $\frac{a}{\dot{\theta}}$ & $\stackrel{\bar{\infty}}{+}$ \\
\hline & 응 & $\begin{array}{c}\infty \\
\infty \\
\infty \\
\infty \\
\alpha \\
d\end{array}$ & 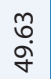 & $\stackrel{\bar{i}}{\bar{i}}$ & $\begin{array}{l}\tilde{D} \\
\ddot{n} \\
\end{array}$ & $\begin{array}{l}\bar{n} \\
\tilde{n} \\
\end{array}$ & $\underset{\bar{\alpha}}{\bar{N}}$ & $\stackrel{\circ}{\aleph}$ & & 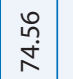 & $\begin{array}{l}\hat{\sigma} \\
\hat{n} \\
\wedge\end{array}$ & 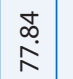 & $\begin{array}{c}\bar{m} \\
\bar{n}\end{array}$ & 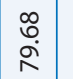 & $\begin{array}{l}\infty \\
\infty \\
\stackrel{+}{\mp}\end{array}$ & $\begin{array}{l}\infty \\
\stackrel{\infty}{2} \\
\stackrel{N}{2}\end{array}$ & $\begin{array}{l}\bar{j} \\
\delta\end{array}$ & 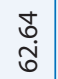 & 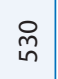 \\
\hline & ๔ & $\begin{array}{l}m \\
\dddot{g} \\
g\end{array}$ & $\bar{E}$ & 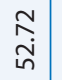 & 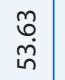 & $\stackrel{m}{\frac{m}{n}}$ & 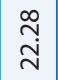 & $\underset{\infty}{\mathfrak{\infty}}$ & & $\begin{array}{l}\stackrel{0}{+} \\
\stackrel{+}{\sim}\end{array}$ & 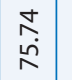 & $\begin{array}{l}\stackrel{a}{\infty} \\
\stackrel{1}{\wedge}\end{array}$ & $\begin{array}{l}\stackrel{m}{n} \\
\\
\end{array}$ & $\begin{array}{c}\hat{N} \\
\stackrel{i}{i}\end{array}$ & $\begin{array}{l}\hat{\tilde{j}} \\
\stackrel{+}{\mp}\end{array}$ & $\begin{array}{l}\text { f } \\
\text { d. }\end{array}$ & \begin{tabular}{l}
$\stackrel{n}{n}$ \\
\multirow{g}{\sigma}{}
\end{tabular} & $\stackrel{\infty}{\stackrel{\infty}{\dot{J}}}$ & $\stackrel{n}{\hat{n}}$ \\
\hline & & 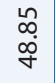 & 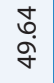 & $\stackrel{\infty}{\underset{i}{n}}$ & 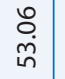 & $\begin{array}{l}\bar{\sigma} \\
\stackrel{\sim}{n}\end{array}$ & $\begin{array}{l}\stackrel{a}{\mathrm{i}} \\
\text { in }\end{array}$ & চু & & $\underset{n}{i n}$ & $\begin{array}{l}\stackrel{N}{m} \\
\stackrel{N}{N}\end{array}$ & 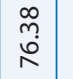 & 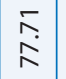 & 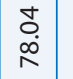 & $\begin{array}{l}\stackrel{a}{0} \\
\stackrel{\dot{n}}{\circ}\end{array}$ & $\begin{array}{l}\stackrel{a}{\infty} \\
\dot{m} \\
\dot{m}\end{array}$ & $\begin{array}{l}\tilde{n} \\
\mathscr{b}\end{array}$ & $\frac{\bar{\sigma}}{\bar{\sigma}}$ & $\stackrel{0}{i n}$ \\
\hline & & $\frac{N}{\stackrel{g}{g}}$ & $\begin{array}{l}\stackrel{\circ}{\circ} \\
\stackrel{+}{\sigma}\end{array}$ & $\stackrel{0}{\stackrel{\sim}{n}}$ & 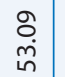 & $\begin{array}{l}\tilde{n} \\
\stackrel{n}{n}\end{array}$ & 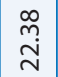 & 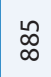 & & $\underset{\substack{\sim\\
}}{ }$ & $\begin{array}{l}\bar{\delta} \\
\stackrel{+}{\perp}\end{array}$ & $\begin{array}{l}\text { ò } \\
\text { مn }\end{array}$ & 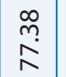 & $\begin{array}{l}\stackrel{\infty}{\wedge} \\
\stackrel{1}{\wedge}\end{array}$ & $\begin{array}{l}m \\
\stackrel{\infty}{0} \\
\stackrel{\varphi}{0}\end{array}$ & $\frac{\dot{m}}{m}$ & $\begin{array}{l}0 \\
\infty \\
\dot{g} \\
\dot{f}\end{array}$ & $\begin{array}{l}\stackrel{0}{\circ} \\
\hat{8}\end{array}$ & 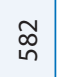 \\
\hline 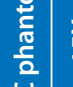 & xִ & $\begin{array}{l}\bar{\infty} \\
\infty \\
\dot{j}\end{array}$ & $\begin{array}{l}\text { in } \\
\stackrel{n}{a} \\
\dot{q}\end{array}$ & $\underset{\sim}{\sim}$ & $\overline{\tilde{n}}$ & $\begin{array}{l}\tilde{O} \\
\stackrel{\sim}{n}\end{array}$ & $\begin{array}{l}\bar{\delta} \\
\underset{\sim}{\mathrm{N}}\end{array}$ & $\stackrel{m}{n}$ & & $\begin{array}{l}\stackrel{\infty}{\mathbb{N}} \\
\stackrel{N}{n}\end{array}$ & $\begin{array}{l}\hat{n} \\
\mathfrak{n}\end{array}$ & $\begin{array}{l}o \\
\dot{o} \\
i\end{array}$ & 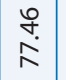 & $\stackrel{\bar{\infty}}{\stackrel{1}{\wedge}}$ & $\begin{array}{l}\mathcal{q} \\
\stackrel{\emptyset}{0}\end{array}$ & $\begin{array}{l}\stackrel{\infty}{N} \\
\stackrel{n}{n}\end{array}$ & $\begin{array}{l}\bar{\delta} \\
\dot{g}\end{array}$ & 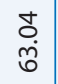 & ฟु \\
\hline & x. & $\begin{array}{l}\stackrel{0}{0} \\
\stackrel{\alpha}{\sigma}\end{array}$ & $\begin{array}{l}\overline{i n} \\
\dot{g}\end{array}$ & $\stackrel{\infty}{\underset{\sim}{n}}$ & $\begin{array}{l}\stackrel{a}{0} \\
\text { in }\end{array}$ & $\begin{array}{l}\tilde{O} \\
\tilde{r} \\
\tilde{n}\end{array}$ & $\stackrel{m}{\underset{\sim}{\sim}}$ & $\stackrel{\infty}{\wedge}$ & & 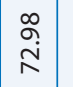 & 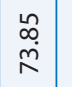 & $\begin{array}{l}o \\
\dot{0} \\
i\end{array}$ & 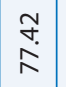 & 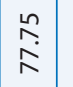 & $\stackrel{m}{\stackrel{m}{\omega}}$ & 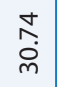 & $\begin{array}{l}\begin{array}{l}J \\
o \\
o\end{array} \\
\end{array}$ & $\frac{\bar{\sigma}}{\bar{\sigma}}$ & $\ddot{\&}$ \\
\hline & త & $\begin{array}{c}\stackrel{n}{\mathfrak{c}} \\
\stackrel{o}{\sigma}\end{array}$ & 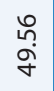 & $\stackrel{\infty}{\underset{i}{n}}$ & $\begin{array}{l}\hat{0} \\
i \\
i n\end{array}$ & 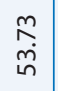 & $\underset{\mathfrak{Z}}{\stackrel{d}{d}}$ & $\stackrel{\sim}{\infty}$ & & 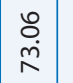 & $\begin{array}{l}\stackrel{a}{\infty} \\
\stackrel{n}{n}\end{array}$ & $\begin{array}{l}\bar{\sigma} \\
\text { hn }\end{array}$ & 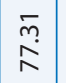 & 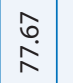 & $\begin{array}{l}\underset{\sim}{n} \\
\stackrel{n}{n}\end{array}$ & $\begin{array}{l}\hat{n} \\
\stackrel{2}{N} \\
\end{array}$ & $\begin{array}{l}\tilde{\sigma} \\
\dot{\gamma} \\
\dot{f}\end{array}$ & $\begin{array}{l}\hat{\lambda} \\
\hat{i}\end{array}$ & 佔 \\
\hline & & $\begin{array}{c}\infty \\
\circ \\
\infty \\
\infty\end{array}$ & $\begin{array}{l}\stackrel{a}{2} \\
\dot{\sigma}\end{array}$ & $\underset{\substack{\sim\\
}}{ }$ & $\begin{array}{l}\stackrel{\bullet}{\circ} \\
\stackrel{\tilde{丶}}{n}\end{array}$ & 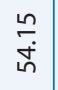 & $\begin{array}{l}\bullet \\
\ddot{j} \\
\end{array}$ & $\stackrel{m}{a}$ & & $\begin{array}{l}\stackrel{t}{0} \\
\dot{R}\end{array}$ & 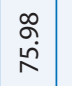 & $\begin{array}{l}\hat{a} \\
\infty \\
\infty \\
\wedge\end{array}$ & $\begin{array}{l}\tilde{m} \\
\text { ğ }\end{array}$ & $\begin{array}{c}\hat{a} \\
\hat{i} \\
\hat{n}\end{array}$ & $\begin{array}{l}\tilde{\Omega} \\
\stackrel{0}{0}\end{array}$ & $\stackrel{\stackrel{\circ}{̣}}{\stackrel{m}{m}}$ & $\begin{array}{c}\hat{n} \\
\hat{\sigma}\end{array}$ & 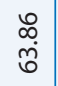 & 会 \\
\hline & & $\begin{array}{c}\infty \\
\stackrel{\infty}{0} \\
\stackrel{b}{q}\end{array}$ & 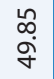 & $\begin{array}{c}\underset{N}{N} \\
\text { in }\end{array}$ & 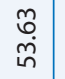 & 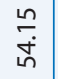 & 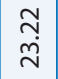 & 会 & & 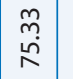 & 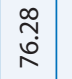 & $\underset{\infty}{\bar{\infty}}$ & 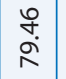 & \begin{tabular}{l}
\multirow{2}{\alpha}{} \\
S.
\end{tabular} & $\begin{array}{l}\stackrel{\infty}{\stackrel{n}{n}} \\
\stackrel{n}{n}\end{array}$ & $\begin{array}{l}\text { ָิ } \\
\text { ஸे }\end{array}$ & $\stackrel{\substack{f \\
\dot{f}}}{ }$ & $\begin{array}{l}\hat{b} \\
\dot{\theta}\end{array}$ & જิ \\
\hline 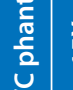 & โิ & $\begin{array}{l}\bar{n} \\
g \\
\sigma\end{array}$ & $\begin{array}{c}m \\
\\
i \\
i n\end{array}$ & $\stackrel{\substack{n \\
\tilde{n}}}{n}$ & 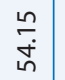 & 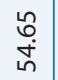 & $\begin{array}{l}\stackrel{0}{\infty} \\
\sim \\
\sim \\
\sim\end{array}$ & ஜे & & 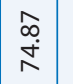 & $\begin{array}{l}\bar{\infty} \\
\stackrel{n}{\wedge}\end{array}$ & $\underset{\infty}{\stackrel{\sigma}{\infty}}$ & $\begin{array}{c}\stackrel{\infty}{n} \\
\stackrel{n}{n}\end{array}$ & $\begin{array}{l}\bar{\infty} \\
\stackrel{\infty}{\wedge}\end{array}$ & $\begin{array}{l}\stackrel{0}{\infty} \\
\stackrel{0}{0}\end{array}$ & $\begin{array}{c}\infty \\
\stackrel{\infty}{m}\end{array}$ & $\begin{array}{l}\infty \\
\stackrel{+}{f} \\
\dot{f}\end{array}$ & $\begin{array}{l}\hat{\infty} \\
\hat{\sigma} \\
\hat{0}\end{array}$ & হ̄ \\
\hline & & 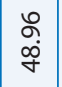 & $\begin{array}{l}\infty \\
\stackrel{\infty}{\sigma} \\
\dot{q}\end{array}$ & $\begin{array}{c}\stackrel{n}{\sim} \\
\stackrel{i}{n}\end{array}$ & 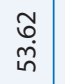 & $\underset{i}{\bar{f}}$ & $\begin{array}{l}\hat{n} \\
\tilde{n} \\
\tilde{n}\end{array}$ & $\stackrel{2}{\curvearrowright}$ & & $\begin{array}{l}\stackrel{n}{\alpha} \\
\dot{\Sigma} \\
\end{array}$ & 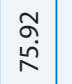 & $\begin{array}{l}\infty \\
\infty \\
\infty \\
\wedge\end{array}$ & $\begin{array}{l}\stackrel{m}{m} \\
\stackrel{n}{n}\end{array}$ & $\begin{array}{c}\bar{\infty} \\
\dot{i} \\
\dot{p}\end{array}$ & $\begin{array}{l}\infty \\
\stackrel{\infty}{0} \\
0\end{array}$ & $\frac{\hat{n}}{\mathfrak{m}}$ & 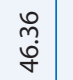 & $\begin{array}{l}\hat{n} \\
\hat{n} \\
\hat{\sigma}\end{array}$ & $\bar{\sim}$ \\
\hline & ช & $\begin{array}{l}\bar{\sigma} \\
\dot{g}\end{array}$ & $\begin{array}{l}n \\
\infty \\
\dot{q} \\
\dot{q}\end{array}$ & $\begin{array}{c}\stackrel{N}{\sim} \\
i n\end{array}$ & 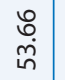 & $\stackrel{m}{\stackrel{m}{\dot{f}}}$ & 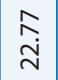 & $\underset{\infty}{\stackrel{\infty}{ }}$ & & $\begin{array}{l}\bar{\delta} \\
\dot{R}\end{array}$ & $\begin{array}{l}\bar{\delta} \\
\dot{0} \\
\wedge\end{array}$ & $\underset{\infty}{\bar{\infty}}$ & $\begin{array}{l}\text { f̊ } \\
\text { ğ }\end{array}$ & $\begin{array}{l}\text { ஃ̊ } \\
\text { న. }\end{array}$ & $\begin{array}{l}\hat{\imath} \\
\stackrel{n}{n}\end{array}$ & $\frac{\vdots}{\sigma}$ & $\begin{array}{c}m \\
\stackrel{f}{f} \\
\hat{f}\end{array}$ & $\begin{array}{l}\text { ๙ } \\
\text { రิ }\end{array}$ & $\stackrel{m}{n}$ \\
\hline 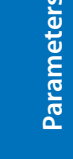 & $\frac{\ddot{\mathrm{g}}}{\frac{\pi}{\sigma}}$ & 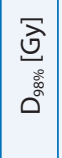 & 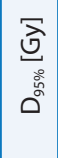 & 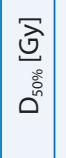 & 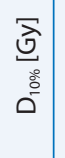 & 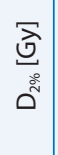 & 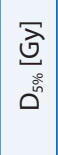 & & $\overrightarrow{\mathrm{g}}$ & 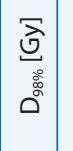 & 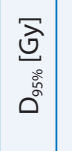 & 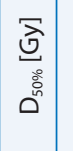 & 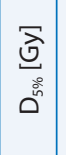 & 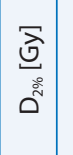 & 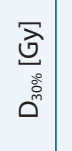 & 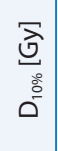 & 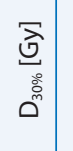 & 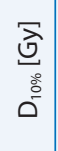 & \\
\hline 总 & 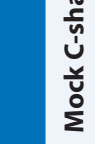 & & & $\begin{array}{l}\vec{z} \\
\vdots \\
y\end{array}$ & & & $\stackrel{0}{\breve{u}}$ & २ & 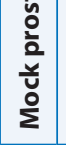 & & & $\begin{array}{l}\vec{z} \\
\text { a } \\
\stackrel{\alpha}{\alpha}\end{array}$ & & & $\begin{array}{l}\frac{\bar{v}}{\frac{0}{0}} \\
\frac{\pi}{0}\end{array}$ & 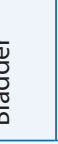 & $\underset{\widetilde{\Xi}}{\varepsilon}$ & E & $\stackrel{\partial}{\Sigma}$ \\
\hline
\end{tabular}


photon beam energies and was comparable with TG $119\left(\mathrm{D}_{95 \%}>75.6 \mathrm{~Gy}\right)$, except for OC phantom using the VMAT technique. The results were tabulated in Table 3B and Table 3D. Dose to the bladder and rectum were within the prescribed limit $\left(\mathrm{D}_{30 \%}<70 \mathrm{~Gy}, \mathrm{D}_{10 \%}<75 \mathrm{~Gy}\right)$ and bladder doses were lesser than all selected studies.

\section{Plan quality indices}

Homogeneity index (HI) was reported and tabulated in Table 7 and Table 8. It was evident that HI was comparatively better for VMAT plans with all the energies in HN PTV and CS PTV and was comparable with IMRT for PR PTV. Similarly, the conformity index (CI) was evaluated and found better with the VMAT technique.

\section{Integral dose of normal tissue}

Dose to normal healthy tissues was calculated and it was found that the IMRT technique deliver more unwanted radiation to body tissues other than tumour target when compared to VMAT plans. It was noted that higher photon energy spills lesser dose to healthy tissues. The dose spillage of 50\% of maximum prescribed dose is shown in Figure 5 and Figure 6.

\section{Variation of plan parameters with standard practice}

Relative measurement was performed for the collected data with standard limiting values prescribed by TG 119 and the results were tabulated in Table 9 and Table 10. It was evident that we had achieved better coverage for the head and neck test target and prostate test target for all the energies in our study. Also the coverage for the multi-target case and Cshaped target was comparable with TG 119 results for both treatment techniques. Maximum dose to the 'cord' was lesser with IMRT but dose to bilateral parotids was almost similar. Dose to the 'centre target' in multi-target case was below the limiting values for the IMRT technique and also for the 'OC phantom' in the VMAT technique. $\mathrm{D}_{10 \%}$ to the 'inferior target' was also not achieved and deviated more for the 'OC phantom' in both techniques and varying energies. Dose received by the 'core' structure was better achieved by the VMAT treatment technique and exceeded for IMRT for all the energies. Dose to the bladder and rectum was within the tolerance, recommended by the TG 119 protocol.

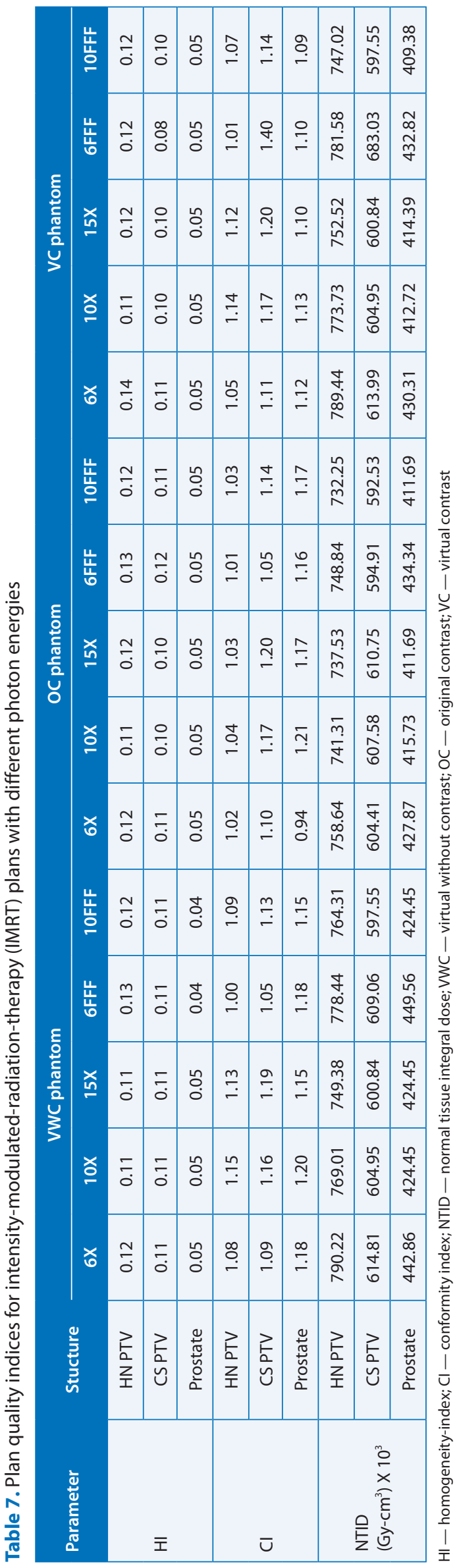




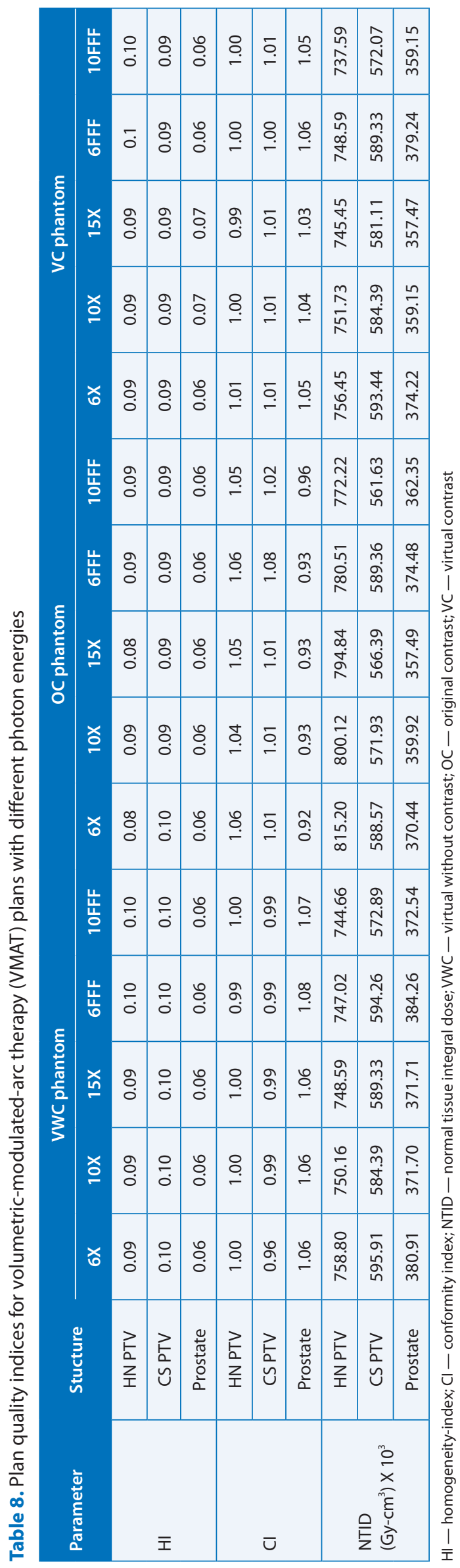

\section{Total monitor units}

Total monitor units were calculated summing monitor units of individual fields of each plan and noted in Table 3. It was evident that requirement of MUs decreases with increasing photon beam energies and it was much less for the VMAT technique, which is shown in Table 11 and Table 12.

\section{Discussion}

Accuracy of treatment delivery depends on accurate dose computation. The use of contrast-enhanced images for treatment planning and delivery of those treatment plans on non-contrast tissues was always a matter of concern for radiotherapy practitioners. The contrast agent includes high- $\mathrm{Z}$ radio-opaque materials which attenuate the $\mathrm{X}$-rays more than the normal tissues. This situation increases the CT number (or Hounsfield Units, i.e. $\mathrm{HU}$ ) and, hence, the electron density in that voxel temporarily which could result in altering photon beam dosimetry $[16,17]$.

We have used test case targets provided by AAPM TG 119 and validated the same with the Eclipse treatment planning system. It was noted in our study that PTV coverage achieved in all the test cases was more than the limiting values and increased with higher photon energy. However, a little difference was observed with contrast-enhanced phantom images for target coverage. Our results are in agreement with results published by Haghparast et al. [18]. Moreover, to relate the photon beam energies with intravenous contrast, Ramm et al. [19] conducted their study. They used a cylinder of barium sulfate (diameter $3 \mathrm{~cm}$ ) in a water phantom and irradiated the same with either $6 \mathrm{MV}$ or $25 \mathrm{MV}$ photon beam energies to evaluate the effect of CT contrast on dose calculation in a three-dimensional planning technique. They concluded that the effect of contrast was very small if the planner increased the number of beams and a dose difference of $2.7 \%$ and $1.8 \%$ was found for $6 \mathrm{MV}$ and $25 \mathrm{MV}$, respectively, for a standard four-field technique due to cylinder of contrast but the dose perturbation was not clinically significant.

Jing et al. [20] highlighted the underestimation of dose due to intravenous contrast was slight and was moderate due to an oral contrast agent. But, Weber et al. [21] reported a slight reduction of dose to the 


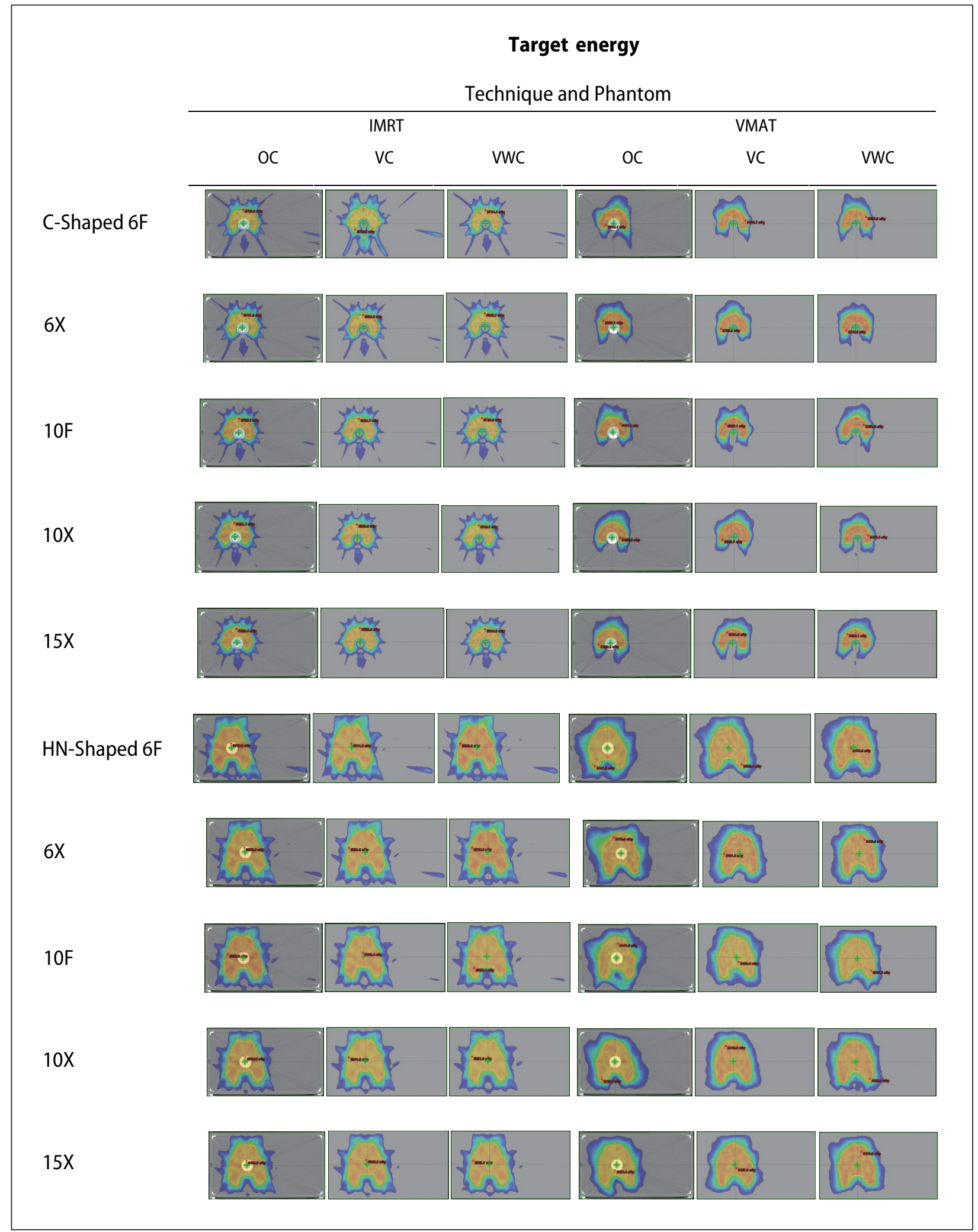

Figure 5. Spillage of 50\% of prescription dose in C-shaped and head-and-neck-shaped test cases (Axial view)

prostate target $(0.03-0.14 \%)$ and rectum $(1.13 \%)$ while studying the effects of bladder opacification on dose calculations with a 6-field conformal plan for prostate cancer patients. In a similar manner, the higher tail-region was reported for the 'central' target in our study. 


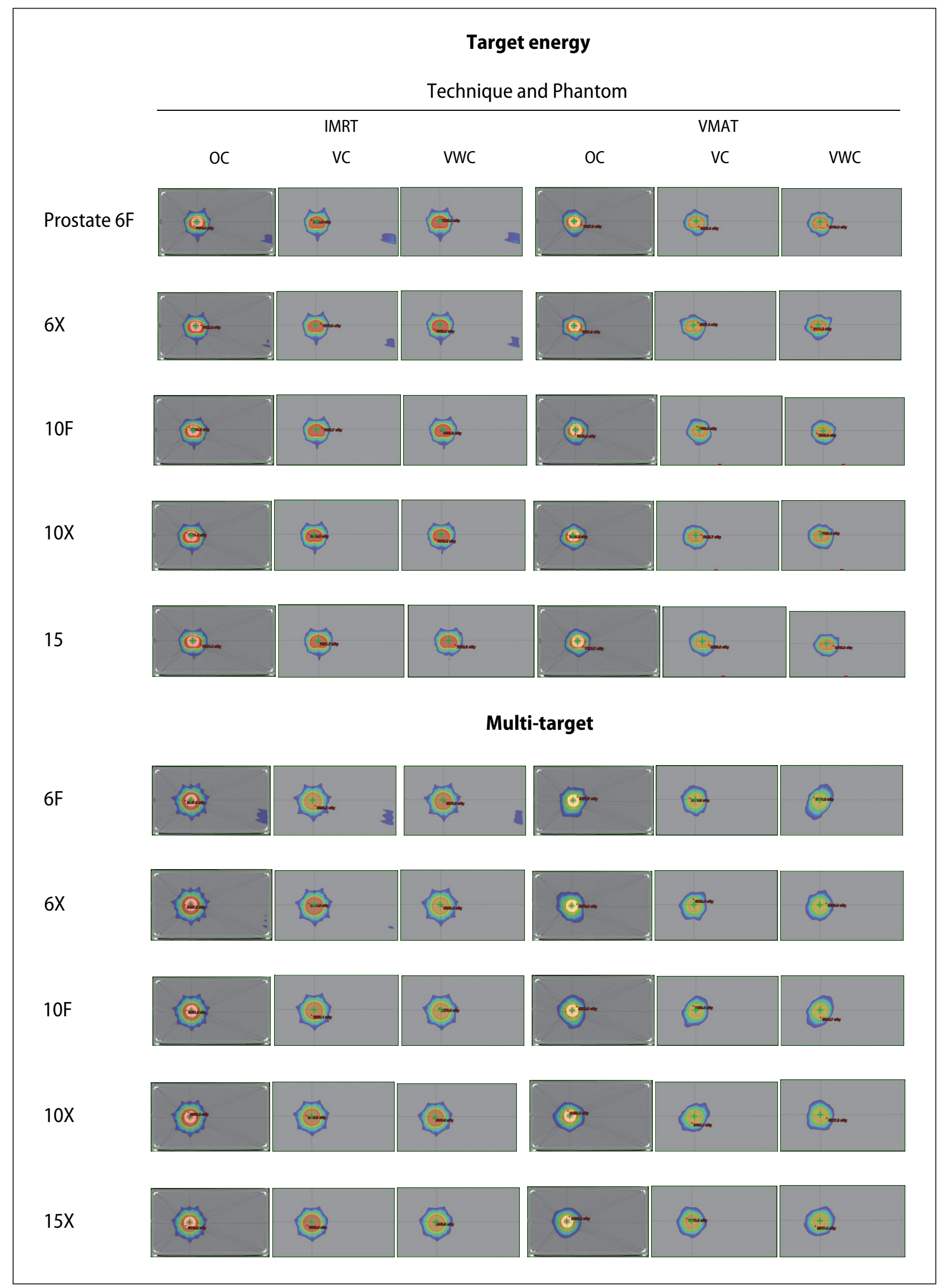

Figure 6. Spillage of 50\% of prescription dose in Prostate-shaped and Multi-target test cases (Axial view) 


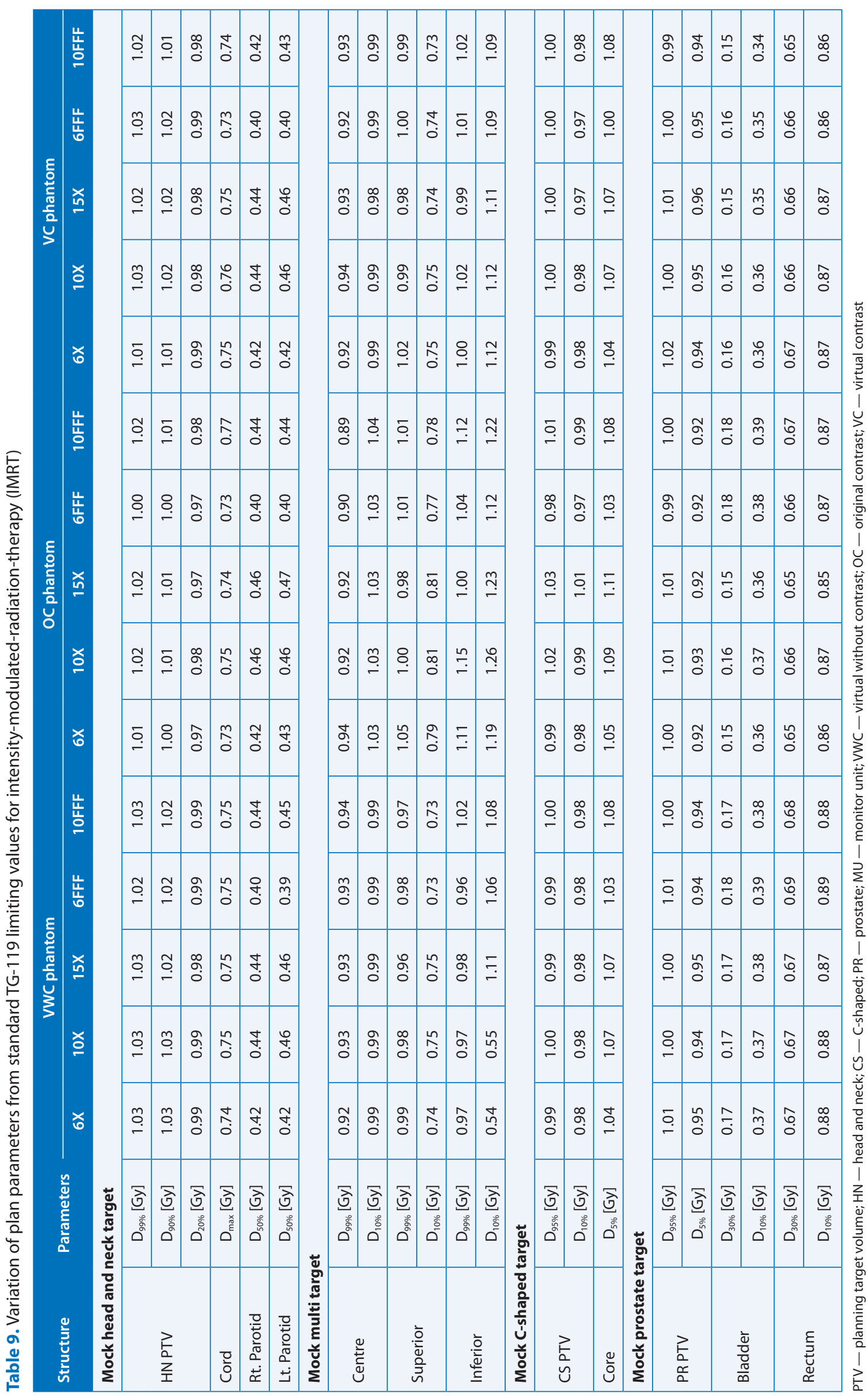




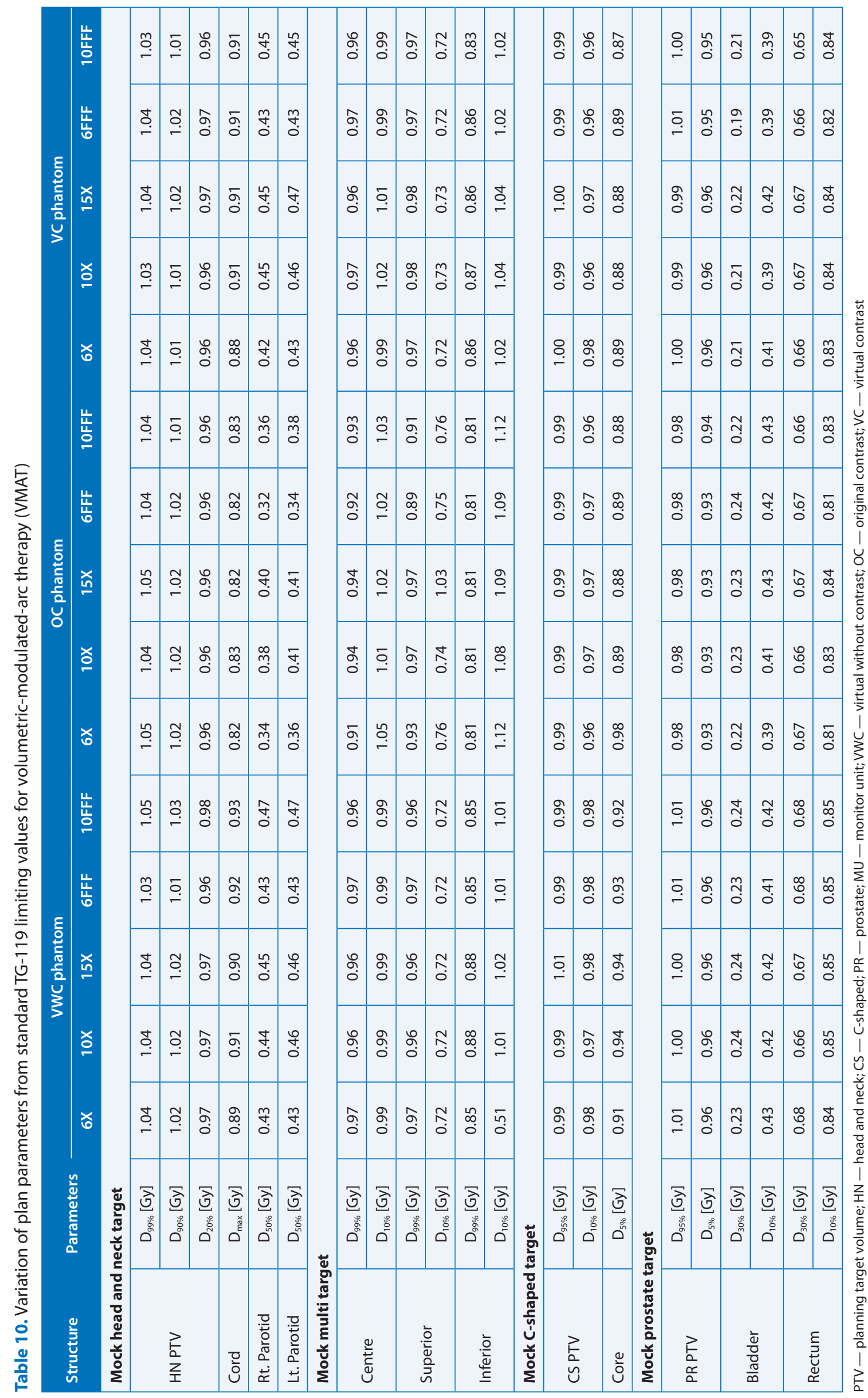




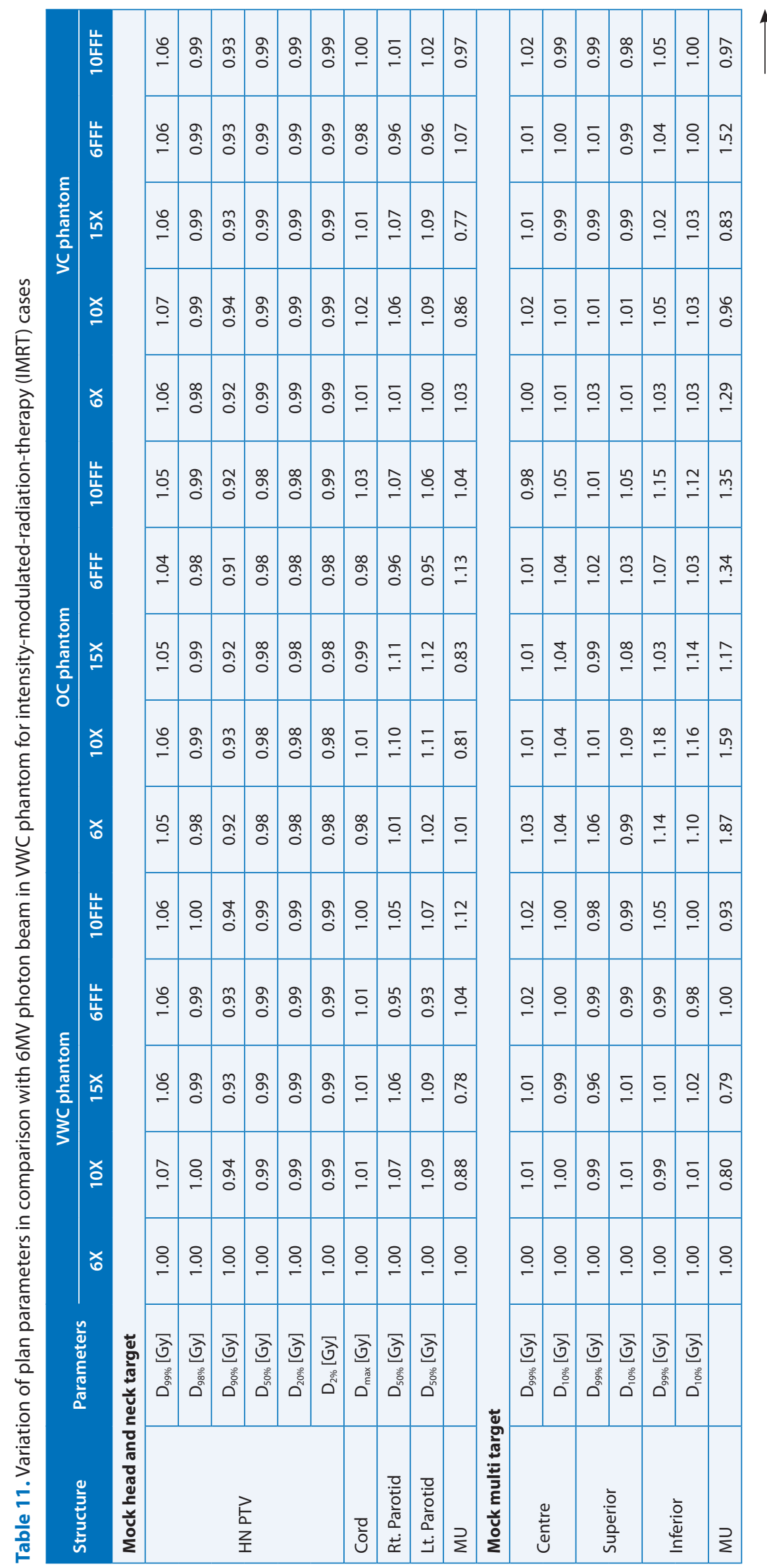




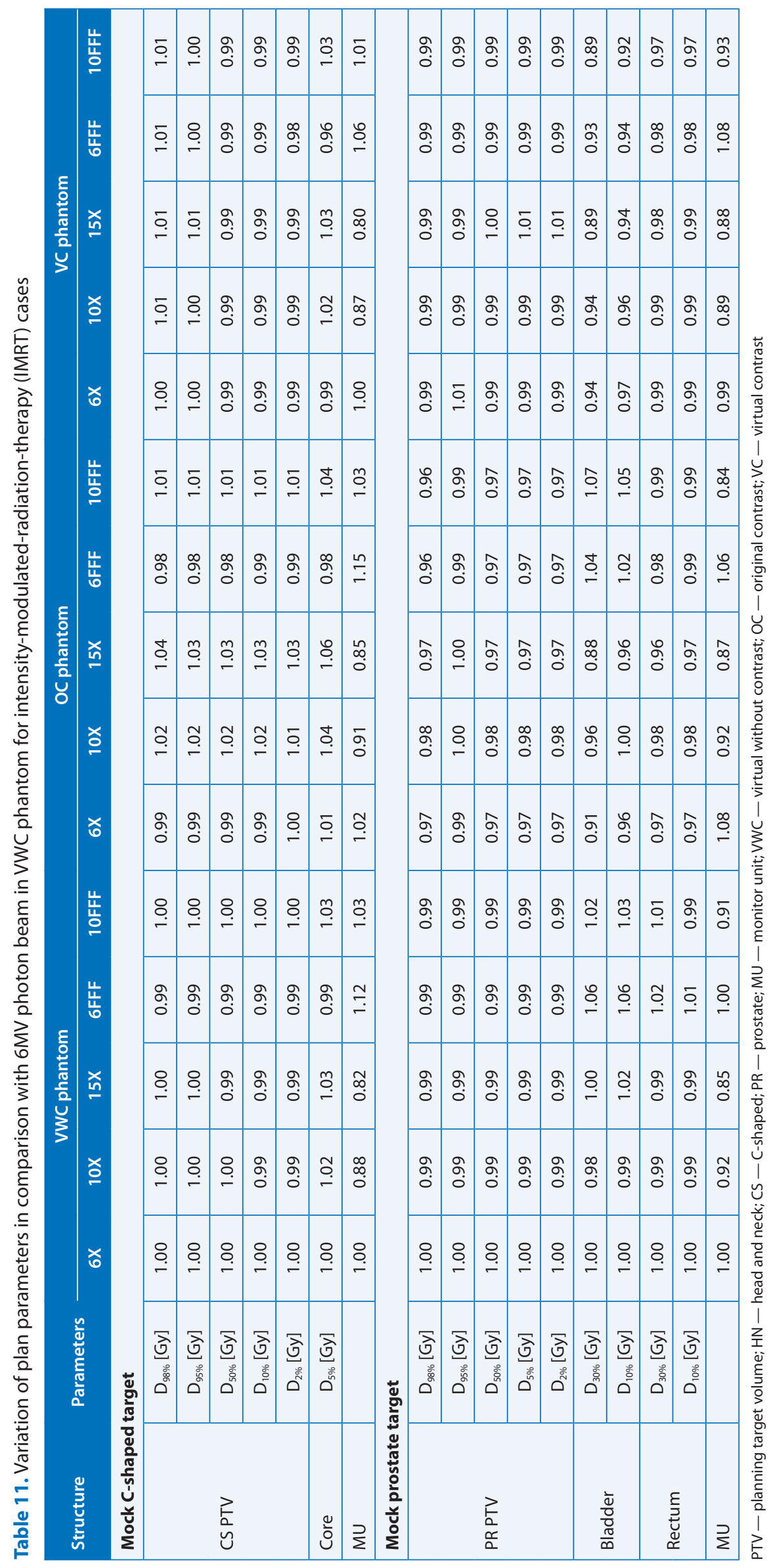




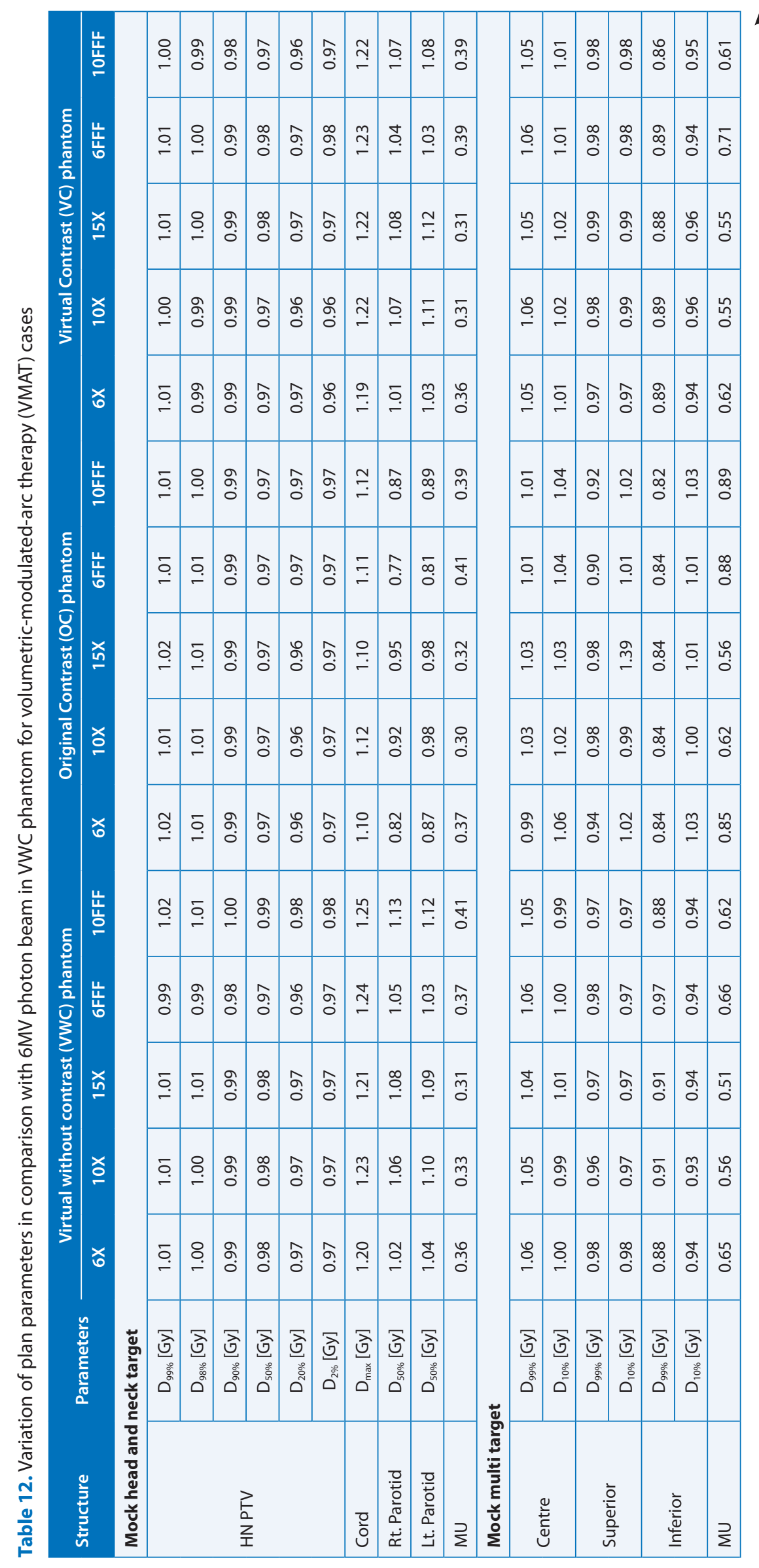




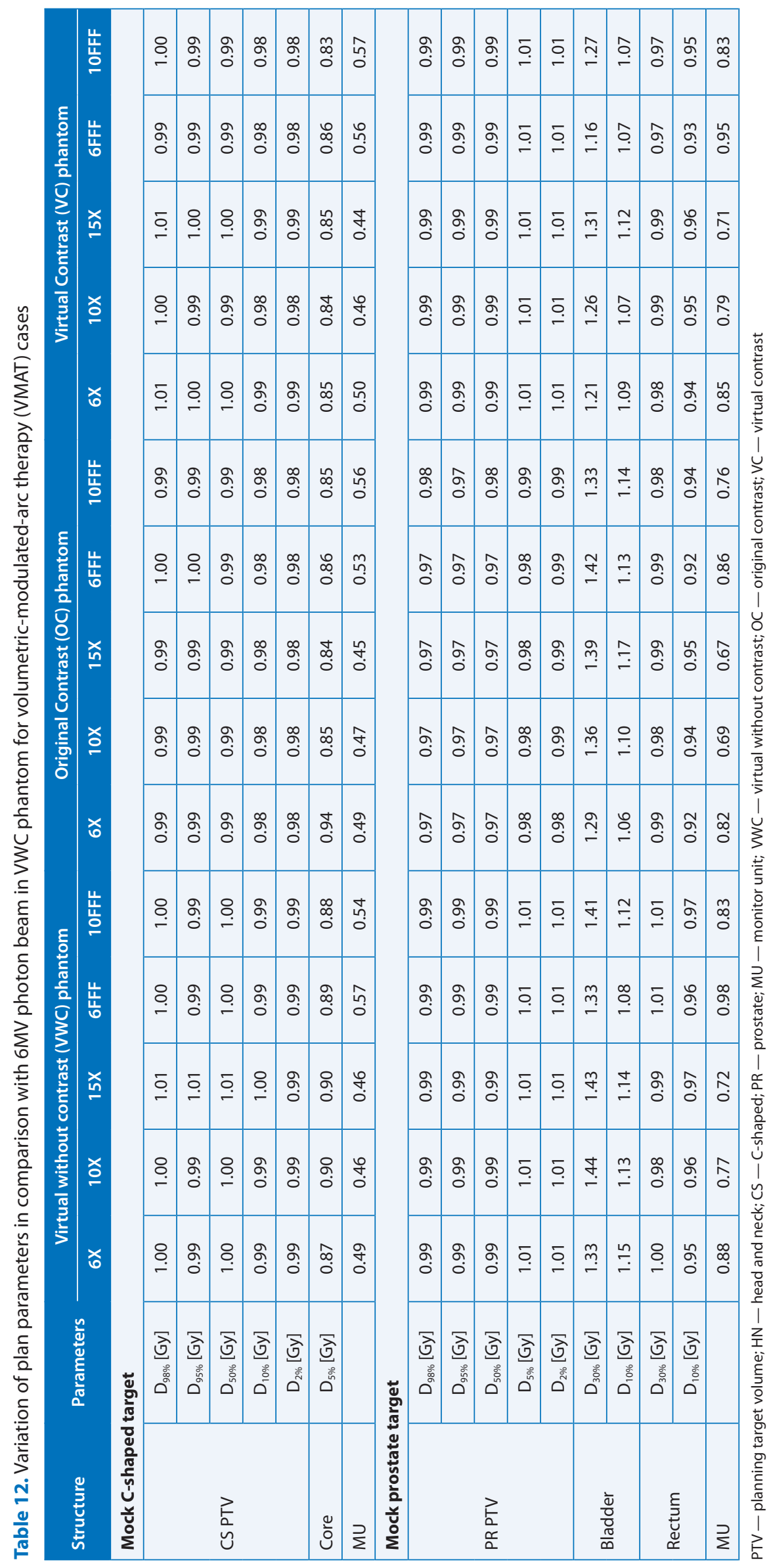


Choi et al. [22] also investigated the effect of intravenous contrast in head and neck patients and reported that IMRT dose distribution remained unchanged and insensitive to contrast media. Additionally, Wertz et al. [23] highlighted the effect of intravenous contrast in skull base tumours at the time of CT simulation and found a maximum change of $36 \mathrm{HU}$ in tumour tissue homogeneity. Maximum dose to the 'core' in the C-shaped target case was improved with VMAT and was increased with higher photon energies due to involvement of exit-dose contribution and increased HU numbers.

As per literature, the choice of beam energy depends on depth of a tumour target due to the spectrum of penetration power and the basic teaching is that the planner should choose higher photon beam energy for deep-seated targets over low-energy photons. But there are discussions among practitioners to select higher photon energies for IMRT and VMAT treatment techniques.

Soderstorm et al. [24] reported that there was no significant difference between $6 \mathrm{MV}$ and $18 \mathrm{MV}$ for radiotherapy treatment while Henry et al. [25] mentioned a clear advantage of dual arc VMAT over IMRT. Similarly, Kumar et al. [26] performed a study using 6 and 10 MV FFF beam and reported the FFFB of $6 \mathrm{MV}$ to be superior as compared to $10 \mathrm{MV}$, for RA planning in case of gynaecological malignancies. Moreover, it offered better $\mathrm{HI}$ and CI values, as well as a lower number of MUs (3.33\%). In addition, it delivered more NTID (4.42\%) for similar target coverage and OAR's sparing. However, Pirzkall et al. [27] demonstrated a significant impact of energy selection on dose distribution. The lesser sparing of the cord in the head and neck test case with VMAT planning might be due to additive contribution of lower dose components in the maximum dose of the structure.

The other aspect of high energy photons related to neutron production was discussed by Gurjar et al. [28] and Kry et al. [29]. The similar results were concluded by Zhai et al. [30] and Yadav et al. [31] and, hence, the use of higher photon beam energies was restricted to conformal radiotherapy planning. It was noted in our study that VMAT planning improved the conformity and homogeneity of the plans due to continuous placement of beams around the targets. Even though Hussein et al. [32] conducted their study and addressed the benefits of IMRT using $15 \mathrm{MV}$ photon beams energy outweighing risk. We found that integral dose to nearby healthy tissues was increased with IMRT plans but higher photon beams delivered less dose to these areas due to their skin-sparing effects. Wonmo et al. [33] also concluded to use 10 MV photons after their known negligible neutron dose equivalent considering their biological and clinical significance. But the present study suggested that the monitor units required for delivering the dose to target with VMAT planning was much lower and further reduced with selection of higher photon energies due to their penetration power in the tissues.

\section{Conclusion}

The contrast-enhanced CT images definitely lower the dose to targets when compared with non-contrast CT structures set. The IMRT or VMAT plans, generated on such enhanced CT images will be delivered with higher doses than evaluated. However, the overdose remains non-significant and not very sensitive to contrast media. Higher photon energies also do not make clinically important changes. As the intravenous contrast is helpful in delineation of targets, we conclude that it is useful to include contrast-enhanced CT images for planning and the dose deviation remains insignificant as the output of treatment delivery.

\section{Authors' contribution}

D.T. - study design, G.Y. - data interpretation, L.K. - data collection, A.D. - statistical analysis, S.T. - literature search, G.K. - manuscript preparation, I.K.W. - literature search, M.G. - data interpretation

\section{Conflict of interest}

No potential conflict of interest related to this article was reported.

None declared.

\section{Funding}

\section{Acknowledgement}

We hereby thank the management of our institute for their continuous support and guidance. 


\section{References}

1. Bray F, Ferlay J, Soerjomataram I, et al. Global cancer statistics 2018: GLOBOCAN estimates of incidence and mortality worldwide for 36 cancers in 185 countries. CA Cancer J Clin. 2018; 68(6): 394-424, doi: 10.3322/ caac.21492, indexed in Pubmed: 30207593.

2. Arbyn $M$, Weiderpass $E$, Bruni $L$, et al. Estimates of incidence and mortality of cervical cancer in 2018: a worldwide analysis. Lancet Glob Health. 2020; 8(2): e191-e203, doi: 10.1016/s2214-109x(19)30482-6, indexed in Pubmed: 31812369.

3. Zelefsky MJ, Lee WR, Zietman A, et al. Evaluation of Adherence to Quality Measures for Prostate Cancer Radiotherapy in the United States: Results from the Quality Research in Radiation Oncology (QRRO) Survey. Pract Radiat Oncol. 2013; 3(1): 2-8, doi: 10.1016/j.prro.2012.01.006, indexed in Pubmed: 23471563.

4. d'Errico F. Dosimetric issues in radiation protection of radiotherapy patients. Radiat Prot Dosimetry. 2006; 118(2): 205-212, doi: $10.1093 / \mathrm{rpd} / \mathrm{ncl} 034$, indexed in Pubmed: 16581918.

5. Uysal B, Beyzadeoğlu M, Sager O, et al. Dosimetric evaluation of intensity modulated radiotherapy and 4-field 3-d conformal radiotherapy in prostate cancer treatment. Balkan Med J. 2013; 30(1): 54-57, doi: 10.5152/balkanmedj.2012.075, indexed in Pubmed: 25207069.

6. Hall E. Intensity-modulated radiation therapy, protons, and the risk of second cancers. Int J Radiat Oncol Biol Phys. 2006; 65(1): 1-7, doi: 10.1016/j.ijrobp.2006.01.027, indexed in Pubmed: 16618572.

7. Kry SF, Salehpour M, Followill DS, et al. The calculated risk of fatal secondary malignancies from intensity-modulated radiation therapy. Int J Radiat Oncol Biol Phys. 2005; 62(4): 1195-1203, doi: 10.1016/j.jijrobp.2005.03.053, indexed in Pubmed: 15990025.

8. Kumar L, Yadav G, Samuvel KR, et al. Dosimetric influence of filtered and flattening filter free photon beam on rapid arc (RA) radiotherapy planning in case of cervix carcinoma. Rep Pract Oncol Radiother. 2017; 22(1): 10-18, doi: 10.1016/j.rpor.2016.09.010, indexed in Pubmed: 27790073.

9. Bhushan M, Yadav G, Tripathi D, et al. Clinical dosimetric impact of AAA and Acuros XB on high-density metallic implants in case of carcinoma cervix. Oncol J India. 2019; 3(2): 28, doi: 10.4103/oji.oji_18_19.

10. Bhushan M, Yadav G, Tripathi D, et al. Dosimetric Analysis of Unflattened (FFFB) and Flattened (FB) Photon Beam Energy for Gastric Cancers Using IMRT and VMAT-a Comparative Study. J Gastrointest Cancer. 2019; 50(3): 408-419, doi: 10.1007/s12029-018-0080-9, indexed in Pubmed: 29520733.

11. Ezzell GA, Burmeister JW, Dogan N, et al. IMRT commissioning: multiple institution planning and dosimetry comparisons, a report from AAPM Task Group 119. Med Phys. 2009; 36(11): 5359-5373, doi: 10.1118/1.3238104, indexed in Pubmed: 19994544.

12. Mynampati DK, Yaparpalvi R, Hong L, et al. Application of AAPM TG 119 to volumetric arc therapy (VMAT). J Appl Clin Med Phys. 2012; 13(5): 3382, doi: 10.1120/jacmp. v13i5.3382, indexed in Pubmed: 22955639.
13. Nithya L, Raj NA, Rathinamuthu S, et al. Analyzing the performance of the planning system by use of AAPMTG 119 test cases. Radiol Phys Technol. 2016; 9(1): 22-29, doi: 10.1007/ s12194-015-0328-z, indexed in Pubmed: 26141766.

14. Avgousti R, Armpilia C, Floros I, et al. Evaluation of intensity modulated radiation therapy delivery system using a volumetric phantom on the basis of the task group 119 report of american association of physicists in medicine. J Med Phys. 2017; 42(1): 33, doi: 10.4103/0971-6203.202419, indexed in Pubmed: 28405106.

15. Kaushik S, Tyagi A, Kumar L, et al. Validation of intensitymodulated radiotherapy commissioning as per recommendations in test plans of the American Association of Physicists in Medicine task group 119 report. Radiat Protect Environ. 2016; 39(3): 138, doi: 10.4103/0972-0464.194960.

16. Yamada S, Ueguchi T, Ogata T, et al. Radiotherapy treatment planning with contrast-enhanced computed tomography: feasibility of dual-energy virtual unenhanced imaging for improved dose calculations. Radiat Oncol. 2014; 9: 168, doi: 10.1186/1748-717X-9-168, indexed in Pubmed: 25070169.

17. Kim HJ, Chang AhR, Park YK, et al. Dosimetric effect of CT contrast agent in CyberKnife treatment plans. Radiat Oncol. 2013; 8: 244, doi: 10.1186/1748-717X-8-244, indexed in Pubmed: 24139405.

18. Heydarheydari S, Farshchian N, Haghparast A. Influence of the contrast agents on treatment planning dose calculations of prostate and rectal cancers. Rep Pract Oncol Radiother. 2016; 21(5): 441-446, doi: 10.1016/j. rpor.2016.04.004, indexed in Pubmed: 27489514.

19. Ramm U, Damrau M, Mose S, et al. Influence of CT contrast agents on dose calculations in a 3D treatment planning system. Phys Med Biol. 2001; 46(10): 2631-2635, doi: 10.1088/0031-9155/46/10/308, indexed in Pubmed: 11686279.

20. Jing $H$, Tian $Y$, Tang $Y u$, et al. Oral contrast agents lead to underestimation of dose calculation in volumetricmodulated arc therapy planning for pelvic irradiation. Chin Med J (Engl). 2020; 133(17): 2061-2070, doi: 10.1097/ CM9.0000000000001025, indexed in Pubmed: 32810050.

21. Weber D, Rouzaud M, Miralbell R. Bladder opacification does not significantly influence dose distribution in conformal radiotherapy of prostate cancer. Radiother Oncol. 2001; 59(1): 95-97, doi: 10.1016/s0167-8140(01)00306-1, indexed in Pubmed: 11295212.

22. Choi Y, Kim JK, Lee HS, et al. Influence of intravenous contrast agent on dose calculations of intensity modulated radiation therapy plans for head and neck cancer. Radiother Oncol. 2006; 81(2): 158-162, doi: 10.1016/j. radonc.2006.09.010, indexed in Pubmed: 17050020.

23. Wertz H, Jäkel O. Influence of iodine contrast agent on the range of ion beams for radiotherapy. Med Phys. 2004; 31(4): 767-773, doi: 10.1118/1.1650871, indexed in Pubmed: 15124994.

24. Söderström S, Eklöf A, Brahme A. Aspects on the optimal photon beam energy for radiation therapy. Acta Oncol. 1999; 38(2): 179-187, doi: 10.1080/028418699431591, indexed in Pubmed: 10227439.

25. Sze HCK, Lee MCH, Hung WM, et al. RapidArc radiotherapy planning for prostate cancer: single-arc and double-arc techniques vs. intensity-modulated radio- 
therapy. Med Dosim. 2012; 37(1): 87-91, doi: 10.1016/j. meddos.2011.01.005, indexed in Pubmed: 21925863.

26. Kumar L, Kishore V, Yadav G, et al. EP-1670: Impact of flatting filter free photon beam on Rapid-arc radiotherapy for gynecological malignancies. Radiother Oncol. 2016; 119: S780, doi: 10.1016/s0167-8140(16)32921-8.

27. Pirzkall A, Carol MP, Pickett B, et al. The effect of beam energy and number of fields on photon-based IMRT for deep-seated targets. Int J Radiat Oncol Biol Phys. 2002; 53(2): 434-442, doi: 10.1016/s0360-3016(02)02750-5, indexed in Pubmed: 12023148.

28. Gurjar O, Jha V, Sharma S. Radiation dose to radiotherapy technologists due to induced activity in high energy medical electron linear accelerators. Radiat Protect Environ. 2014; 37(1): 25, doi: 10.4103/0972-0464.146460.

29. Kry SF, Salehpour M, Followill DS, et al. Out-of-field photon and neutron dose equivalents from step-andshoot intensity-modulated radiation therapy. Int J Radiat Oncol Biol Phys. 2005; 62(4): 1204-1216, doi: 10.1016/j. ijrobp.2004.12.091, indexed in Pubmed: 15990026.

30. Zhai DY, Yin Y, Gong GZ, et al. RapidArc radiotherapy for whole pelvic lymph node in cervical cancer with 6 and
$15 \mathrm{MV}$ : a treatment planning comparison with fixed field IMRT. J Radiat Res. 2013; 54(1): 166-173, doi: 10.1093/jrr/ rrs066, indexed in Pubmed: 23283869.

31. Yadav G, Bhushan M, Dewan A, et al. Dosimetric influence of photon beam energy and number of arcs on volumetric modulated arc therapy in carcinoma cervix: A planning study. Rep Pract Oncol Radiother. 2017; 22(1): 1-9, doi: 10.1016/j.rpor.2016.09.002, indexed in Pubmed: 27790072.

32. Hussein $M$, Aldridge $S$, Guerrero Urbano $T$, et al. The effect of 6 and 15 MV on intensity-modulated radiation therapy prostate cancer treatment: plan evaluation, tumour control probability and normal tissue complication probability analysis, and the theoretical risk of secondary induced malignancies. Br J Radiol. 2012; 85(1012): 423-432, doi: 10.1259/bjr/24514638, indexed in Pubmed: 22010028.

33. Sung W, Park JM, Choi CH, et al. The effect of photon energy on intensity-modulated radiation therapy (IMRT) plans for prostate cancer. Radiat Oncol J. 2012; 30(1): 27-35, doi: 10.3857/roj.2012.30.1.27, indexed in Pubmed: 23120741. 